\title{
CONSERVATIVE GALERKIN METHODS FOR DISPERSIVE HAMILTONIAN PROBLEMS
}

\author{
JAMES JACKAMAN AND TRISTAN PRYER
}

\begin{abstract}
An energy conservative discontinuous Galerkin scheme for a generalised third order KdV type equation is designed. Based on the conservation principle, we propose techniques that allow for the derivation of optimal a priori bounds for the linear KdV equation and a posteriori bounds for the linear and modified KdV equation. Extensive numerical experiments showcasing the good long time behaviour of the scheme are summarised which are in agreement with the analysis proposed.
\end{abstract}

\section{INTRODUCTION}

Hamiltonian partial differential equations (PDEs) arise naturally from a variety of physically motivated application areas, with decisive examples including meteorological, as exemplified by the semi-geostrophic equations [4], and oceanographical, such as the Korteweg-de Vries (KdV) and nonlinear Schrödinger equations 41. The KdV and nonlinear Schrödinger equations are particularly special examples, in that they are bi-Hamiltonian [36. This means they have two different Hamiltonian formulations which, in turn, is one way to understand the notion of integrability of these problems.

Conservative schemes for Hamiltonian ordinary differential equations (ODEs) are, by now, relatively well understood, see [8, 9, 11, 25, 35, c.f.]. Typically numerical schemes designed for this class of problem have some property of the ODE built into them, for example preservation of the Hamiltonian or the underlying symplectic form, are classified as geometric integrators.

In this contribution, we consider a family of Hamiltonian PDEs that generalise the famous defocusing modified KdV equation

$$
u_{t}-\frac{3}{2} u^{2} u_{x}+u_{x x x}=0
$$

where the sub-indices denote partial differentiation with respect to the corresponding independent variable. This equation has numerous applications including fluid dynamics and plasma physics [1. The Korteweg third order term, as well as the non-linearity, can cause issues in the numerical treatment of this problem. In particular, the combination of these two terms cause significant trouble in the design of numerical methods that are optimally convergent in the function approximation sense.

In previous numerical studies of the scalar $\mathrm{KdV}$ and modified $\mathrm{KdV}$ equations [46,47, c.f.], it has been observed that classical finite volume and discontinuous Galerkin $(\mathrm{dG})$ schemes with "standard" numerical fluxes introduce numerical artefacts. Consequently the long-term dynamics of solutions may be destroyed by the addition of artificial diffusion. Such diffusion in a given scheme endows it with desirable stability properties, however, it typically destroys all information in the long-term dynamics of the system through smearing of solutions.

Hamiltonian problems are inherently conservative in the sense that the underlying Hamiltonian is conserved over time. Such equations may have additional structures which manifest themselves through additional conserved quantities. In particular, for the KdV equation mass and momentum are such quantities. In [10,29] the authors propose and analyse a $\mathrm{dG}$ method for generalised KdV equations. The method itself is very carefully designed to be conservative, in that the invariant corresponding to the momentum is inherited by the discretisation. This naturally yields $L^{2}$ stability for the numerical method along with extremely good long time dynamics. In the scalar case one may also design schemes that conserve the energy itself [27, 45, however, it does not seem possible to design schemes to conserve more than two of these invariants. This is, in part, due to the invariants differing in order of nonlinearity. Other promising mechanisms to approximate such problems include those based on the Fokas transform [31] and the Chebfun package [15] both of which experimentally has extremely good long time properties as the schemes naturally inherit many of the properties of the PDE up to small precision. Alternatively, one may aim to preserve the multi-symplectic structure of the KdV equation, which has proven successful with Preissmann box schemes [3,48 resulting in discretisations possessing desirable qualitative behaviour. The study of multi-symplectic schemes is a fertile area of research with finite element discretisations utilising this structure currently being developed, for example [12,39].

J.J. was partially supported through a PhD scholarship awarded by the "EPSRC Centre for Doctoral Training in the Mathematics of Planet Earth at Imperial College London and the University of Reading" EP/L016613/1, and the Canadian Research Chairs and NSERC Discovery grant programs. T.P. was partially supported through the EPSRC grant EP/P000835/1 and the Newton Fund grant 261865400. Both authors would additionally like to acknowledge the support of the Isaac Newton Institute for Mathematical Sciences, Cambridge through the EPSRC grant EP/K032208/1. 
Our goal in this work is the derivation of Galerkin discretisations aimed at preserving the underlying algebraic properties satisfied by the PDE system whilst avoiding the introduction of stabilising diffusion terms. Our schemes are therefore consistent with (one of) the Hamiltonian formulation(s) of the original problem which physically represents energy. We note that our approach is not an adaptation of entropy conserving schemes developed for systems of conservation laws, rather we study the algebraic properties of the PDE and formulate the discretisation to inherit this specific structure. Our methods are of arbitrarily high order accuracy in space, extendable to arbitrarily high order in time, and provide relevant approximations free from numerical artefacts. Similar techniques have proven useful in the study of dispersive phase flow problems [19,21] and we anticipate they will be extremely useful in dynamic model adaptivity [24].

To highlight the good behaviour of the scheme we propose, we give an a priori error analysis for the linear problem showing optimal error bounds in the energy norm, under the assumption of smoothness in the initial conditions. Further, we give an a posteriori error analysis making use of a hybrid framework consisting of elliptic reconstruction techniques [32, 33, 38, together with those developed for hyperbolic conservation laws [20] to allow us to derive optimal a posteriori error bounds in the energy norm. Note that the arguments we use are quite different to that of [29] where the authors construct a dispersive reconstruction to allow for a posteriori control in $L^{2}$.

The remainder of this work is set out as follows: In Section 2 we introduce notation, the model problem and some of its properties. We also give some exact solutions to the problem that will be useful in numerical benchmarking. In Section 3 we reformulate of the problem through the introduction of an auxiliary variable, introduced to allow for a simple construction of the numerical scheme. We propose a spatial discretisation based on discontinuous Galerkin finite elements, show it is conservative, well posed, and give an a priori analysis yielding optimal error bounds in the energy norm. In Section 4 we give an a posteriori analysis making use of elliptic reconstruction techniques. In Section 5 we state a fully discrete scheme, show it is conservative and summarise extensive numerical experiments validating the analysis done in Section 3 4

\section{Problem Setup}

In this section we formulate the model problem, fix notation and give some basic assumptions. We describe some known results and history of the defocusing generalised Korteweg-de Vries equation, highlighting the Hamiltonian structure of the equation. We show that the underlying Hamiltonian structure naturally yields an induced stability of the solutions to the PDE system and give a summary of some exact solutions for specific non-linearities.

Throughout this work we consider the (1+1)-dimensional dispersive Cauchy problem

$$
\begin{array}{rl}
u_{t}-f^{\prime}(u)_{x}+u_{x x x}=0 & x \in S^{1}, \quad t>0 \\
u(x, 0)=u^{0}(x) & x \in S^{1}
\end{array}
$$

with periodic boundary conditions over the unit interval $S^{1}:=[0,1]$ with endpoints being identified with one another. The non-linearity is polynomial, specifically

$$
f(u)=\alpha u^{m} \text { for } 2 \leq m \in \mathbb{Z}, 0<\alpha \in \mathbb{R} .
$$

Examples of the PDE include the linear KdV equation

$$
u_{t}-\alpha u_{x}+u_{x x x}=0,
$$

and the mKdV equation

$$
u_{t}-\alpha u^{2} u_{x}+u_{x x x}=0 .
$$

These equations are the focus of the analysis in this work.

Notice the sign in front of the first order term. In an analogy to the nonlinear Schrödinger equation we refer to as a defocusing equation, with the focusing equations having the opposing sign on the non-linearity.

Proposition 2.1. The dispersive problem (2.1) has the following three base invariants:

$$
\begin{aligned}
\frac{\mathrm{d}}{\mathrm{d} t} F_{-1}(u) & :=\frac{\mathrm{d}}{\mathrm{d} t} \int_{S^{1}} u \mathrm{~d} x=0 \\
\frac{\mathrm{d}}{\mathrm{d} t} F_{0}(u) & :=\frac{\mathrm{d}}{\mathrm{d} t} \int_{S^{1}} \frac{1}{2} u^{2} \mathrm{~d} x=0 \\
\frac{\mathrm{d}}{\mathrm{d} t} F_{1}(u) & :=\frac{\mathrm{d}}{\mathrm{d} t} \int_{S^{1}} \frac{1}{2} u_{x}^{2}+f(u) \mathrm{d} x=0 .
\end{aligned}
$$

Throughout this work we denote the standard Lebesgue spaces by $L^{p}(\omega), 1 \leq p \leq \infty, \omega \subset \mathbb{R}$, with corresponding norms $\|\cdot\|_{L^{p}(\omega)}$. Let also $H^{s}(\omega)$, be the Hilbertian Sobolev space of index $s \in \mathbb{R}$ of real-valued functions defined on $\omega \subset \mathbb{R}^{d}$, 
constructed via standard interpolation and/or duality procedures, along with the corresponding norm and semi-norm

$$
\begin{aligned}
&\|u\|_{W^{k, p}\left(S^{1}\right)}:= \begin{cases}\left(\sum_{|\alpha| \leq k}\left\|\mathrm{D}^{\alpha} u\right\|_{L^{p}\left(S^{1}\right)}^{p}\right)^{1 / p} & \text { if } p \in[1, \infty) \\
\sum_{|\alpha| \leq k}\left\|\mathrm{D}^{\alpha} u\right\|_{L^{\infty}\left(S^{1}\right)} & \text { if } p=\infty\end{cases} \\
&|u|_{W^{k, p}\left(S^{1}\right)}:=\left\|\mathrm{D}^{k} u\right\|_{L^{p}\left(S^{1}\right)}
\end{aligned}
$$

respectively. We also make use of the following notation for time dependent Sobolev (Bochner) spaces:

$$
\begin{gathered}
C^{i}\left(0, T ; H^{k}\left(S^{1}\right)\right):=\left\{u:[0, T] \rightarrow H^{k}\left(S^{1}\right): u \text { and temporal derivatives up to } i \text {-th order are continuous }\right\}, \\
L^{\infty}\left(0, T ; H^{k}\left(S^{1}\right)\right):=\left\{u:[0, T] \rightarrow H^{k}\left(S^{1}\right):{\left.\operatorname{ess} \sup _{t \in[0, T]}\|u(t)\|_{H^{k}\left(S^{1}\right)}<\infty\right\} .},\right.
\end{gathered}
$$

Under some regularity assumptions on the initial condition one can make use of semi-group techniques to show the following:

Proposition 2.2 ([2.1) is well posed [30]). Given $u^{0} \in H^{s}\left(S^{1}\right)$, with $s \geq 3$. Then (2.1) has a unique solution with

$$
u \in C^{0}\left(0, T ; H^{s}\left(S^{1}\right)\right) \cap C^{1}\left(0, T ; H^{s-3}\left(S^{1}\right)\right),
$$

for arbitrary $T>0$.

Proposition 2.3 (Pointwise solution control). Notice that energy conservation

$$
\frac{\mathrm{d}}{\mathrm{d} t} \int_{S^{1}} u_{x}^{2}+f(u) \mathrm{d} x=0
$$

and mass conservation

$$
\frac{\mathrm{d}}{\mathrm{d} t} \int_{S^{1}} u \mathrm{~d} x=0
$$

immediately shows that,

$$
\|u\|_{C^{i}\left(0, T ; H^{1}\left(S^{1}\right)\right)} \leq C\left(\left\|u_{x}^{0}\right\|_{L^{2}\left(S^{1}\right)}^{2}+\left\|u^{0}\right\|_{L^{m}\left(S^{1}\right)}^{m}\right)^{1 / 2}
$$

for some constant $C>0$. Since $H^{1}\left(S^{1}\right) \subset L^{\infty}\left(S^{1}\right)$ we see

$$
\sup _{t \in[0, \infty]}\|u(t)\|_{L^{\infty}\left(S^{1}\right)} \leq C\left(\left\|u_{x}^{0}\right\|_{L^{2}\left(S^{1}\right)}^{2}+\left\|u^{0}\right\|_{L^{m}\left(S^{1}\right)}^{m}\right)^{1 / 2} .
$$

Proposition 2.4 (Exact solution to the linear problem). Let $f(u)=\frac{1}{2} u^{2}$, then under the ansatz that $u(t, x)=u(\xi)$, with $\xi=c\left(x+\left(1+c^{2}\right) t\right)$ we find that

$$
u(x, t)=C_{1} \sin (\xi)+C_{2} \cos (\xi),
$$

solves (2.1) where $c=2 l \pi$ for $l \in \mathbb{Z}$ and $C_{1}, C_{2}$ denote real constants. Due to the linear nature of the problem any linear combination of (2.15) for various attainable parameter values is also a solution.

Proposition 2.5 (Exact solution to the nonlinear problem). With $f(u)=6 u^{3}$, then it can be shown that the position solution

$$
u(x, t)=\frac{c}{2} \operatorname{csch}\left(-\frac{c^{1 / 2}(x-c t)}{2}\right)^{2}
$$

formally solves (2.1). It is well-known that one can map solutions from the defocusing $m K d V$ equation to solutions to the KdV equation employing the Miura transform. Although, it is worth noting that it is not possible to get smooth, non-singular position solutions of the defocusing $m K d V$ through inverse scattering techniques because of the singularity that is inherent in its Darboux transformation. For $f(u)=\frac{1}{4} u^{4}$, one can, however find kink

$$
u(x, t)=(3 c)^{1 / 2} \tanh \left(\frac{(2 c)^{1 / 2}}{2}(x+c t)\right)
$$

and anti-kink solutions

$$
u(x, t)=-(3 c)^{1 / 2} \tanh \left(\frac{(2 c)^{1 / 2}}{2}(x+c t)\right),
$$

that are smooth, but are not periodic. To establish periodic, smooth exact solutions, one must examine Jacobi elliptic functions 42]. Let $\operatorname{sn}(x, k)$ denote that Jacobi elliptic function with modulus $k \in[0,1)$, then, with $f(u)=\frac{1}{2} u^{4}$, a solution is given by [13]

$$
u(x, t)=k \operatorname{sn}\left(x+\left(k^{2}+1\right) t, k\right) .
$$




\section{DisCRETISATION AND A PRIORI ANALYSIS}

We consider the approximation of (2.1) by a semi-discrete discontinuous Galerkin scheme. Let $0=x_{0}<x_{1}<$ $\cdots<x_{N}=1$ be a partition of the periodic domain $S^{1}$. We denote $I_{j}=\left[x_{j}, x_{j+1}\right]$ to be the $j$-th sub-interval and let $h_{j}:=x_{j+1}-x_{j}$ be its size. We denote the piece-wise constant mesh-size function $h: S^{1} \rightarrow[0, \infty)$ where $\left.h\right|_{I_{j}}=h_{j}$. For the purposes of this work, we will assume that $\max \left(h_{j} N\right) \leq C$ for some $C>0$. For $q \geq 1$ let $\mathbb{P}^{q}(I)$ be the space of polynomials of degree less than or equal to $q$ on $I$, then we denote

$$
\mathbb{V}_{q}:=\left\{g: S^{1} \rightarrow \mathbb{R}:\left.g\right|_{I_{j}} \in \mathbb{P}^{q}\left(I_{j}\right) \text { for } j=0, \ldots, N-1\right\} .
$$

In addition, we define jump and average operators by

$$
\begin{aligned}
& \llbracket g \rrbracket_{j}:=g\left(x_{j}^{-}\right)-g\left(x_{j}^{+}\right):=\lim _{s \searrow 0} g\left(x_{j}-s\right)-\lim _{s \searrow 0} g\left(x_{j}+s\right), \\
& \{g\}_{j}:=\frac{1}{2}\left(g\left(x_{j}^{-}\right)+g\left(x_{j}^{+}\right)\right):=\frac{1}{2}\left(\lim _{s \searrow 0} g\left(x_{j}-s\right)+\lim _{s \searrow 0} g\left(x_{j}+s\right)\right)
\end{aligned}
$$

where the periodic boundary conditions are accounted for by $\llbracket g \rrbracket_{0}:=g\left(x_{N}^{-}\right)-g\left(x_{0}^{+}\right)$and $\{g\}_{0}:=\frac{1}{2}\left(g\left(x_{N}^{-}\right)+g\left(x_{0}^{+}\right)\right)$. Throughout this work we will use the convention that $C>0$ denotes a generic constant which may depend on $q$, the ratio of concurrent cell sizes and non-linearity degree $m$, but is independent of $h$ and the exact solution $u$.

We will examine semi-discrete numerical schemes which are based on the following reformulation of (2.1) using an auxiliary variable $v$

$$
\begin{aligned}
u_{t}+v_{x} & =0 \\
v+f^{\prime}(u)-u_{x x} & =0 .
\end{aligned}
$$

The purpose of this variable becomes apparent in the discretisation of (2.1). Indeed, $v$ is deliberately chosen as the first variation of the energy functional, $F_{1}$. We note that a similar numerical procedure was applied to a regularised elastodynamics problem in [22,23. We will begin by introducing some projection operators and describe some of their properties we will make use of throughout this work.

Definition 3.1 ( $L^{2}$ projection operator and properties). We define the $L^{2}$ projection operator $\mathscr{P}_{h}: L^{2}\left(S^{1}\right) \rightarrow \mathbb{V}_{q}$ by requiring

$$
\sum_{j=0}^{N-1} \int_{x_{j}}^{x_{j+1}} \mathscr{P}_{h}(w) \Phi \mathrm{d} x=\sum_{j=0}^{N-1} \int_{x_{j}}^{x_{j+1}} w \Phi \mathrm{d} x \quad \forall \Phi \in \mathbb{V}_{q} .
$$

When $w \in H^{q+1}\left(S^{1}\right)$, the following approximation properties hold

$$
\left\|w-\mathscr{P}_{h}(w)\right\|_{L^{2}\left(S^{1}\right)} \leq C h^{q+1}|w|_{H^{q+1}\left(S^{1}\right)} .
$$

Definition 3.2 (Discrete gradients $\mathscr{G}_{h}$ and properties). The discrete gradient operator $\mathscr{G}_{h}: \prod_{j=0}^{N-1} H^{1}\left(I_{j}\right) \rightarrow \mathbb{V}_{q}$ is defined by

$$
\sum_{j=0}^{N-1} \int_{x_{j}}^{x_{j+1}} \mathscr{G}_{h}(w) \Psi \mathrm{d} x=\sum_{j=0}^{N-1} \int_{x_{j}}^{x_{j+1}} w_{x} \Psi \mathrm{d} x-\sum_{j=0}^{N-1} \llbracket w \rrbracket_{j}\{\Psi\}_{j} \quad \forall \Psi \in \mathbb{V}_{q} .
$$

It can be seen from the definition that these operators satisfy a discrete integration by parts, that is for $W, \Psi \in \mathbb{V}_{q}$

$$
\sum_{j=0}^{N-1} \int_{x_{j}}^{x_{j+1}} \mathscr{G}_{h}(W) \Psi \mathrm{d} x=-\sum_{j=0}^{N-1} \int_{x_{j}}^{x_{j+1}} W \mathscr{G}_{h}(\Psi) \mathrm{d} x .
$$

Definition 3.3 (Interior penalty bilinear form and properties). We define the interior penalty bi-linear form for $w, \psi \in$ $\prod_{j=0}^{N-1} H^{2}\left(I_{j}\right)$ as

$$
\mathscr{A}_{h}(w, \psi):=\sum_{j=0}^{N-1}\left(\int_{x_{j}}^{x_{j+1}} w_{x} \psi_{x} \mathrm{~d} x-\llbracket w \rrbracket_{j}\left\{\psi_{x}\right\}_{j}-\llbracket \psi \rrbracket_{j}\left\{w_{x}\right\}_{j}+\sigma\{h\}_{j}^{-1} \llbracket w \rrbracket_{j} \llbracket \psi \rrbracket_{j}\right),
$$

for some $\sigma \gg 1$. Note that this is symmetric, that is

$$
\mathscr{A}_{h}(w, \psi)=\mathscr{A}_{h}(\psi, w),
$$

and a consistent representation of the Laplacian so for $u \in H^{2}\left(S^{1}\right)$ we have

$$
\mathscr{A}_{h}(u, \psi)=\int_{S^{1}}-u_{x x} \psi \mathrm{d} x .
$$


Semi discrete scheme. With these definitions in hand we are now in a position to state the semi discretisation of (3.3). This is to seek $U \in C^{1}\left([0, T), \mathbb{V}_{q}\right)$ and $V \in C^{0}\left([0, T), \mathbb{V}_{q}\right)$ such that

$$
\begin{aligned}
\int_{S^{1}} U_{t} \Phi+\mathscr{G}_{h}(V) \Phi \mathrm{d} x & =0 \quad \forall \Phi \in \mathbb{V}_{q}, t \in[0, T] \\
\int_{S^{1}}\left(V+f^{\prime}(U)\right) \Psi+\mathscr{A}_{h}(U, \Psi) & =0 \quad \forall \Psi \in \mathbb{V}_{q}, t \in[0, T] \\
U(0) & =\mathscr{P}_{h}\left(u^{0}\right) .
\end{aligned}
$$

Proposition 3.4 (Conservativity of discrete invariants). Solutions $U \in C^{1}\left([0, T), \mathbb{V}_{q}\right)$ and $V \in C^{0}\left([0, T), \mathbb{V}_{q}\right)$ to the discrete scheme (3.11) satisfy conservation of mass,

$$
\frac{\mathrm{d}}{\mathrm{d} t} F_{-1}(U)=\frac{\mathrm{d}}{\mathrm{d} t}\left(\int_{S^{1}} U \mathrm{~d} x\right)=0
$$

and the discrete energy identity

$$
\frac{\mathrm{d}}{\mathrm{d} t} F_{1, h}(U):=\frac{\mathrm{d}}{\mathrm{d} t}\left(\frac{1}{2} \mathscr{A}_{h}(U, U)+\int_{S^{1}} f(U) \mathrm{d} x\right)=0 .
$$

Proof. We see (3.12) by taking $\Phi=1$ in (3.11) and utilising the definition of $\mathscr{G}_{h}$. For (3.13) we explicitly compute the time derivative and use the symmetry of $\mathscr{A}_{h}(\cdot, \cdot)$. Hence

$$
\frac{\mathrm{d}}{\mathrm{d} t} F_{1, h}(U)=\mathscr{A}_{h}\left(U, U_{t}\right)+\int_{S^{1}} f^{\prime}(U) U_{t} \mathrm{~d} x=-\int_{S^{1}} V U_{t} \mathrm{~d} x=\int_{S^{1}} \mathscr{G}_{h}(V) V \mathrm{~d} x=0,
$$

as required.

Corollary 3.5 (Pointwise discrete solution control). Through similar arguments as Proposition 2.3 we have that

$$
\sup _{t \in[0, T]}\|U(t)\|_{L^{\infty}\left(S^{1}\right)} \leq C .
$$

Lemma 3.6 (Existence and uniqueness to the discrete scheme (3.11)). For given initial data $U(0) \in \mathbb{V}_{q}$ the ODE system (3.11) has a unique solution with $\left.U \in C^{1}\left((0, T), \mathbb{V}_{q}\right)\right)$.

Proof. We begin by eliminating the auxiliary variable by writing (3.11) in primal form. To that end, we define the discrete Laplacian $A_{h}: \mathbb{V}_{q} \rightarrow \mathbb{V}_{q}$ such that for any fixed $\Psi \in \mathbb{V}_{q}$

$$
\int_{S^{1}}-A_{h}(\Psi) \Phi \mathrm{d} x=\mathscr{A}_{h}(\Psi, \Phi) \quad \forall \Phi \in \mathbb{V}_{q} .
$$

Then (3.11) can be written as

$$
\int_{S^{1}}\left(U_{t}-\mathscr{G}_{h}\left(\mathscr{P}_{h}\left(f^{\prime}(U)\right)-A_{h}(U)\right)\right) \Phi=0,
$$

which allows us to interpret the scheme point-wise as an ODE

$$
U_{t}=: y^{\prime}(t)=F(y(t)):=\mathscr{G}_{h}\left(\mathscr{P}_{h}\left(f^{\prime}(U)\right)-A_{h}(U)\right) .
$$

In view of inverse estimates and the stability of the $L^{2}$ projector we see that $F$ is continuous. Further, through the conservativity of the scheme from Proposition 3.4 we see that $y$ remains in a bounded set, which depends upon the initial data, as long as a classical solution to (2.1) exists, irrespective of the non-linearity. That is, for $y(0) \in K \subset \mathbb{V}_{q}, y(t) \in K$ for all $t$. Further the Jacobian

$$
\mathrm{D} F(y)(z)=\mathscr{G}_{h}\left(\mathscr{P}_{h}\left(f^{\prime \prime}(y) z\right)-A_{h}(z)\right)
$$

is a uniformly bounded operator. We may now invoke the Picard-Lindelöf theorem yielding a global solution.

A priori error analysis. We dedicate the rest of this section to the a priori error analysis of the scheme (3.11) for the linear problem. We proceed by making use of the discrete stability framework satisfied by the approximation, introducing appropriate projection operators and defining the mesh dependent norms for our analysis.

Lemma 3.7 (Perturbed error equation). Let $(U, V)$ be a solution of (3.11) and let

$$
(\widetilde{U}, \widetilde{V}) \in C^{1}\left([0, T), \mathbb{V}_{q}\right) \times C^{0}\left([0, T), \mathbb{V}_{q}\right)
$$

satisfy the following perturbed problem

$$
\begin{aligned}
\int_{S^{1}} \widetilde{U}_{t} \Phi+\mathscr{G}_{h}(\widetilde{V}) \Phi \mathrm{d} x=\int_{S^{1}}-E^{u} \Phi \mathrm{d} x & \forall \Phi \in \mathbb{V}_{q} \\
\int_{S^{1}} \widetilde{V} \Psi+f^{\prime}(U)-f^{\prime}\left(\theta^{u}\right) \Psi \mathrm{d} x+\mathscr{A}(\widetilde{U}, \Psi)=\int_{S^{1}}-E^{v} \Psi \mathrm{d} x & \forall \Psi \in \mathbb{V}_{q},
\end{aligned}
$$


where $E^{u}, E^{v} \in C^{0}\left([0, T), \mathbb{V}_{q}\right)$ represent discrete residuals and $\theta^{u}=U-\widetilde{U}$. Then, with $\theta^{v}=V-\widetilde{V}$ we have

$$
\frac{\mathrm{d}}{\mathrm{d} t} F_{1, h}\left(\theta^{u}\right)=\int_{S^{1}} f^{\prime}\left(\theta^{u}\right) E^{u}-E^{v} \mathscr{G}_{h}\left(\theta^{v}\right) \mathrm{d} x+\mathscr{A}_{h}\left(\theta^{u}, E^{u}\right) .
$$

Proof. To begin we note that a discrete error equation is given by taking the difference of (3.11) and (3.21) yielding

$$
\begin{aligned}
\int_{S^{1}} \theta_{t}^{u} \Phi+\mathscr{G}_{h}\left(\theta^{v}\right) \Phi \mathrm{d} x & =\int_{S^{1}} E^{u} \Phi \mathrm{d} x \\
\int_{S^{1}} \theta^{v} \Psi+f^{\prime}\left(\theta^{u}\right) \Psi \mathrm{d} x+\mathscr{A}_{h}\left(\theta^{u}, \Psi\right) & =\int_{S^{1}} E^{v} \Psi \mathrm{d} x .
\end{aligned}
$$

Explicitly computing the time derivative

$$
\frac{\mathrm{d}}{\mathrm{d} t} F_{1, h}\left(\theta^{u}\right)=\frac{\mathrm{d}}{\mathrm{d} t}\left(\frac{1}{2} \mathscr{A}_{h}\left(\theta^{u}, \theta^{u}\right)+\int_{S^{1}} f\left(\theta^{u}\right) \mathrm{d} x\right)=\mathscr{A}_{h}\left(\theta^{u}, \theta_{t}^{u}\right)+\int_{S^{1}} f^{\prime}\left(\theta^{u}\right) \theta_{t}^{u} \mathrm{~d} x .
$$

Now making use of (3.23) with $\Psi=\theta_{t}^{u}$ we see

$$
\frac{\mathrm{d}}{\mathrm{d} t} F_{1, h}\left(\theta^{u}\right)=\int_{S^{1}}\left(E^{v}-\theta^{v}\right) \theta_{t}^{u} \mathrm{~d} x
$$

Again using (3.23), this time with $\Phi=E^{v}-\theta^{v}$, we see

$$
\begin{aligned}
\frac{\mathrm{d}}{\mathrm{d} t} F_{1, h}\left(\theta^{u}\right) & =\int_{S^{1}}\left(E^{v}-\theta^{v}\right)\left(E^{u}-\mathscr{G}_{h}\left(\theta^{v}\right)\right) \mathrm{d} x \\
& =\int_{S^{1}} E^{v} E^{u}-\theta^{v} E^{u}-E^{v} \mathscr{G}_{h}\left(\theta^{v}\right) \mathrm{d} x,
\end{aligned}
$$

where we have used skew-symmetry of $\mathscr{G}_{h}$. Further, again by (3.23) with $\Psi=E^{u}$, we have

$$
\frac{\mathrm{d}}{\mathrm{d} t} F_{1, h}\left(\theta^{u}\right)=\int_{S^{1}} f^{\prime}\left(\theta^{u}\right) E^{u}-E^{v} \mathscr{G}_{h}\left(\theta^{v}\right) \mathrm{d} x+\mathscr{A}_{h}\left(\theta^{u}, E^{u}\right),
$$

as required.

Lemma 3.8 (Projection operator $\mathscr{S}_{h}$ and error control). Suppose $v \in H^{q+2}\left(S^{1}\right)$. Let the polynomial degree $q$ be even, the mesh-size be uniform, and the number of elements in the mesh, $N$, be odd. Then, there exists a uniquely defined projection operator, $\mathscr{S}_{h}(v) \in \mathbb{V}_{q}$, satisfying

$$
\begin{aligned}
\int_{S^{1}} \mathscr{S}_{h}(v) \Phi \mathrm{d} x & =\int_{S^{1}} v \Phi \mathrm{d} x \quad \forall \Phi \in \mathbb{V}_{q-1} \\
\left\{\mathscr{S}_{h}(v)\right\}_{j} & =v\left(x_{j}\right) \quad \forall j \in[0, N] .
\end{aligned}
$$

Furthermore, the following error bound holds:

$$
\left\|v-\mathscr{S}_{h}(v)\right\|_{L^{2}\left(S^{1}\right)}+\left\|v_{x}-\mathscr{G}_{h}\left(\mathscr{S}_{h}(v)\right)\right\|_{L^{2}\left(S^{1}\right)} \leq C h^{q+1}\|v\|_{W^{q+2, \infty}\left(S^{1}\right)} .
$$

Proof. We begin by introducing a related projection. Let $\mathscr{T}_{h}(v) \in \mathbb{V}_{q}$ be defined by

$$
\begin{aligned}
\int_{S^{1}} \mathscr{T}_{h}(v) \Phi \mathrm{d} x & =\int_{S^{1}} v \Phi \mathrm{d} x \quad \forall \Phi \in \mathbb{V}_{q-1} \\
\mathscr{T}_{h}(v)\left(x_{j}^{+}\right) & =v\left(x_{j}\right) \quad \forall j \in[0, N] .
\end{aligned}
$$

Notice that, in contrast to $\mathscr{S}_{h}$, the projector $\mathscr{T}_{h}$ has "one-sided" boundary conditions, which means it is locally constructed. It is uniquely defined and has the approximation property

$$
\left\|v-\mathscr{T}_{h}(v)\right\|_{L^{\infty}\left(I_{j}\right)} \leq C h^{q+1}\|v\|_{W^{q+1, \infty}\left(I_{j}\right)} .
$$

Proofs of this can be found in [23, Lem 8].

The remainder of the proof takes inspiration from [10, Prop 3.1]. To show properties for $\mathscr{S}_{h}$ we will consider the error $e=\mathscr{S}_{h}(v)-\mathscr{T}_{h}(v)$. Notice that this satisfies the error relations

$$
\begin{aligned}
\int_{x_{j}}^{x_{j+1}} e \Phi \mathrm{d} x & =0 \quad \forall \Phi \in \mathbb{P}^{q-1}\left(I_{j}\right), j=0, \ldots, N-1 \\
\{e\}_{j} & =\frac{1}{2}\left(v\left(x_{j}\right)-\mathscr{T}_{h}\left(x_{j}^{-}\right)\right) \text {for } j=0, \ldots, N-1 .
\end{aligned}
$$

Let $l_{k} \in \mathbb{P}^{k}(-1,1)$ denote the $k^{t h}$ Legendre polynomial on $(-1,1)$ and $l_{j, k} \in \mathbb{P}^{k}\left(I_{j}\right)$ the transformation to $I_{j}$ given by

$$
l_{j, k}(x)=l_{k}\left(2\left(x-x_{j}\right) / h_{j}-1\right) .
$$


We can then write

$$
e_{j}(x):=\left.e(x)\right|_{I_{j}}=\sum_{k=0}^{q} \alpha_{j, k} l_{j, k}(x) \text { for } j=0, \ldots, N-1
$$

Since

$$
\int_{I_{j}} l_{j, k}(x) l_{j, m}(x) \mathrm{d} x=0 \quad \forall k \neq m
$$

by the orthogonality condition on $e$ we can conclude $\alpha_{j, k}=0$ for $k=0, \ldots, q-1$ and hence

$$
e_{j}(x)=\alpha_{j, q} l_{j, q}(x) \text {. }
$$

Now making use of the second condition

$$
\begin{aligned}
\{e\}_{j} & =\frac{1}{2}\left(e\left(x_{j}^{-}\right)+e\left(x_{j}^{+}\right)\right) \\
& =\frac{1}{2}\left(e_{j-1}\left(x_{j}^{-}\right)+e_{j}\left(x_{j}^{+}\right)\right) \\
& =\frac{1}{2}\left(\alpha_{j-1, q} l_{j-1, q}\left(x_{j}\right)+\alpha_{j, q} l_{j, q}\left(x_{j}\right)\right) \\
& =\frac{1}{2}\left(\alpha_{j-1, q}+(-1)^{q} \alpha_{j, q}\right),
\end{aligned}
$$

through properties of the Legendre polynomials. Taking into account all edge contributions, this yields a linear system for the coefficients $\alpha_{j, q}$,

$$
\left[\begin{array}{ccccc}
(-1)^{q} & 0 & 0 & \ldots & 1 \\
1 & (-1)^{q} & 0 & \ldots & 0 \\
0 & 1 & (-1)^{q} & \cdots & 0 \\
\vdots & \vdots & \vdots & \ddots & \vdots \\
0 & 0 & 0 & \ldots & (-1)^{q}
\end{array}\right]\left[\begin{array}{c}
\alpha_{0, q} \\
\alpha_{1, q} \\
\alpha_{2, q} \\
\vdots \\
\alpha_{N-1, q}
\end{array}\right]=\left[\begin{array}{c}
v\left(x_{0}\right)-\mathscr{T}_{h}\left(x_{0}^{-}\right) \\
v\left(x_{1}\right)-\mathscr{T}_{h}\left(x_{1}^{-}\right) \\
v\left(x_{2}\right)-\mathscr{T}_{h}\left(x_{2}^{-}\right) \\
\vdots \\
v\left(x_{N-1}\right)-\mathscr{T}_{h}\left(x_{N-1}^{-}\right)
\end{array}\right]
$$

This system is invertible only when $N$ is odd and $q$ is even which also ensures uniqueness of the projector $\mathscr{S}_{h}(v)$.

Now solving this system, we have

$$
\left[\begin{array}{c}
\alpha_{0, q} \\
\alpha_{1, q} \\
\alpha_{2, q} \\
\vdots \\
\alpha_{N-1, q}
\end{array}\right]=\frac{1}{2}\left[\begin{array}{cccccc}
1 & 1 & -1 & \ldots & 1 & -1 \\
-1 & 1 & 1 & \ldots & -1 & 1 \\
1 & -1 & 1 & \ldots & 1 & -1 \\
\vdots & \vdots & \vdots & \ddots & \ddots & \vdots \\
1 & -1 & 1 & \ldots & -1 & 1
\end{array}\right]\left[\begin{array}{c}
v\left(x_{0}\right)-\mathscr{T}_{h}\left(x_{0}^{-}\right) \\
v\left(x_{1}\right)-\mathscr{T}_{h}\left(x_{1}^{-}\right) \\
v\left(x_{2}\right)-\mathscr{T}_{h}\left(x_{2}^{-}\right) \\
\vdots \\
v\left(x_{N-1}\right)-\mathscr{T}_{h}\left(x_{N-1}^{-}\right)
\end{array}\right]
$$

From (3.31) we know

$$
\left|v\left(x_{j}\right)-\mathscr{T}_{h}\left(x_{j}^{-}\right)\right| \leq C h^{q+1}\|v\|_{W^{q+1, \infty}\left(I_{j}\right)} \text { for } j=0, \ldots, N-1 .
$$

To conclude we invoke the results of [10, Prop 3.2] that states

$$
\left|v\left(x_{i}\right)-\mathscr{T}_{h}\left(x_{i}^{-}\right)-v\left(x_{i+1}\right)+\mathscr{T}_{h}\left(x_{i+1}^{-}\right)\right| \leq C h^{q+2}\|v\|_{W^{q+2, \infty}\left(I_{i} \cup I_{i+1}\right)} \text { for } i=0, \ldots, N-2 .
$$

Appropriately extending the result over the periodic boundary we have

$$
\left|\alpha_{j, q}\right| \leq C h^{q+1}|u|_{W^{q+2, \infty}\left(S^{1}\right)},
$$

by (3.31). This yields $L^{\infty}\left(S^{1}\right)$ control on the error and $L^{2}\left(S^{1}\right)$ control as a consequence. For the gradient bound, note through the definition of $\mathscr{G}_{h}$ we have

$$
\begin{aligned}
\left\|\mathscr{P}_{h}\left(v_{x}\right)-\mathscr{G}_{h}\left(\mathscr{S}_{h}(v)\right)\right\|_{L^{2}\left(S^{1}\right)} & =\sup _{\phi \in L^{2},\|\phi\| \leq 1} \int_{S^{1}}\left(\mathscr{P}_{h}\left(v_{x}\right)-\mathscr{G}_{h}\left(\mathscr{S}_{h}(v)\right)\right) \phi \\
& =\sup _{\phi \in L^{2},\|\phi\| \leq 1} \int_{S^{1}}\left(v_{x}-\mathscr{S}_{h}\left(\mathscr{S}_{h}(v)\right)\right) \mathscr{P}_{h}(\phi) \\
& =\sup _{\phi \in L^{2},\|\phi\| \leq 1}-\int_{S^{1}}\left(v-\mathscr{S}_{h}(v)\right)\left(\mathscr{P}_{h}(\phi)\right)_{x} \mathrm{~d} x+\sum_{j=0}^{N-1}\left\{v-\mathscr{S}_{h}(v)\right\}_{j} \llbracket \mathscr{P}_{h}(\phi) \rrbracket_{j} \\
& =0,
\end{aligned}
$$

by the definition of $\mathscr{S}_{h}$. Hence $\mathscr{P}_{h}\left(v_{x}\right)=\mathscr{G}_{h}\left(\mathscr{S}_{h}(v)\right)$ and the result follows through the approximation properties of the $L^{2}$ projection. 
Remark 3.9 (Restrictions of Lemma 3.8). The a priori analysis that follows has restrictions that stem from defining the projection operator $\mathscr{S}_{h}$, the mesh-size should be uniform, although it is possible to relax this condition, the polynomial degree should be even and the number of grid points odd. The reason is to have access to an operator that is consistent with our discretisation of the discrete derivative operator. Numerically, we observe optimal convergence of the method regardless of the polynomial degree and the number of grid points.

Note that similar conditions are required for the dispersive projection operator in [10]. These conditions can be removed using an L-dG approach 47]. We have chosen to pursue this approach to enable us to use elliptic reconstruction techniques for the a posteriori analysis following in Section 4.

Definition 3.10 (Mesh dependent norms). Let $V(h):=\mathbb{V}_{q}+H^{2}\left(S^{1}\right)$. We define two mesh dependent $H^{1}$ - like norms as

$$
\begin{aligned}
\|W\|_{V(h)}^{2} & :=\sum_{j=0}^{N-1}\left(\left\|W_{x}\right\|_{L^{2}\left(I_{j}\right)}^{2}+\left\|h W_{x x}\right\|_{L^{2}\left(I_{j}\right)}^{2}+\{h\}_{j}^{-1} \llbracket W \rrbracket_{j}^{2}\right) \\
\|W\|_{\mathbb{V}_{q}}^{2} & :=\sum_{j=0}^{N-1}\left(\left\|W_{x}\right\|_{L^{2}\left(I_{j}\right)}^{2}+\{h\}_{j}^{-1} \llbracket W \rrbracket_{j}^{2}\right) .
\end{aligned}
$$

When the penalty parameter, $\sigma$, is chosen large enough, the interior penalty bi-linear form (3.8) is coercive over $\mathbb{V}_{q}$ and continuous over $V(h)$ with respect to the norm $\|\cdot\|_{V(h)}$, that is

$$
\begin{aligned}
C_{c}\|W\|_{V(h)}^{2} & \leq \mathscr{A}_{h}(W, W) \quad \forall W \in \mathbb{V}_{q} \\
\mathscr{A}_{h}(w, \phi) & \leq C_{b}\|w\|_{V(h)}\|\phi\|_{V(h)} \quad \forall w, \phi \in V(h) .
\end{aligned}
$$

For $W \in \mathbb{V}_{q}$ the two norms are equivalent and in particular

$$
\|W\|_{\mathbb{V}_{q}} \leq\|W\|_{V(h)} .
$$

We will make use of $\|\cdot\|_{V(h)}$ for a priori analysis and $\|\cdot\|_{\mathbb{V}_{q}}$ and $\|\cdot\|_{V(h)}$ for a posteriori analysis.

Lemma 3.11 (Inconsistent Ritz projector $\mathscr{R}_{h}$ and error control). Let the conditions in Lemma 3.8 hold. For $u \in$ $W^{q+3, \infty}\left(S^{1}\right)$, let $\mathscr{R}_{h}(u) \in \mathbb{V}_{q}$ satisfy

$$
\mathscr{A}_{h}\left(\mathscr{R}_{h}(u), \Phi\right)+\int_{S^{1}} \mathscr{R}_{h}(u) \Phi \mathrm{d} x=\mathscr{A}_{h}(u, \Phi)+\int_{S^{1}}\left(u+v-\mathscr{S}_{h}(v)\right) \Phi \mathrm{d} x
$$

Then, for h small enough

$$
\left\|u-\mathscr{R}_{h}(u)\right\|_{L^{2}\left(S^{1}\right)}+h\left\|u-\mathscr{R}_{h}(u)\right\|_{V(h)} \leq C h^{q+1}\|u\|_{W^{q+3, \infty}\left(S^{1}\right)} .
$$

Proof. To show (3.48) we note that through the definition (3.47) we have the orthogonality result

$$
\mathscr{A}_{h}\left(\mathscr{R}_{h}(u)-u, \Phi\right)+\int_{S^{1}}\left(\mathscr{R}_{h}(u)-u\right) \Phi \mathrm{d} x=\int_{S^{1}}\left(v-\mathscr{S}_{h}(v)\right) \Phi \mathrm{d} x \quad \forall \Phi \in \mathbb{V}_{q} .
$$

Hence we have, for any $W \in \mathbb{V}_{q}$

$$
\begin{aligned}
C_{c}\left\|W-\mathscr{R}_{h}(u)\right\|_{V(h)}^{2}+\left\|W-\mathscr{R}_{h}(u)\right\|_{L^{2}\left(S^{1}\right) \leq}^{2} & \mathscr{A}_{h}\left(W-\mathscr{R}_{h}(u), W-\mathscr{R}_{h}(u)\right)+\int_{S^{1}}\left(W-\mathscr{R}_{h}(u)\right)\left(W-\mathscr{R}_{h}(u)\right) \mathrm{d} x \\
= & \mathscr{A}_{h}\left(W-u, W-\mathscr{R}_{h}(u)\right)+\int_{S^{1}}(W-u)\left(W-\mathscr{R}_{h}(u)\right) \mathrm{d} x \\
& +\mathscr{A}_{h}\left(u-\mathscr{R}_{h}(u), W-\mathscr{R}_{h}(u)\right)+\int_{S^{1}}\left(u-\mathscr{R}_{h}(u)\right)\left(W-\mathscr{R}_{h}(u)\right) \mathrm{d} x \\
= & \mathscr{A}_{h}\left(W-u, W-\mathscr{R}_{h}(u)\right)+\int_{S^{1}}(W-u)\left(W-\mathscr{R}_{h}(u)\right) \mathrm{d} x \\
& \quad+\int_{S^{1}}\left(\mathscr{S}_{h}(v)-v\right)\left(W-\mathscr{R}_{h}(u)\right) \mathrm{d} x \\
\leq & \frac{1}{2}\left(\frac{C_{b}^{2}}{C_{c}}\|W-u\|_{V(h)}^{2}+C_{c}\left\|W-\mathscr{R}_{h}(u)\right\|_{V(h)}^{2}+\|W-u\|_{L^{2}\left(S^{1}\right)}^{2}\right) \\
& \left.+2\left\|W-\mathscr{R}_{h}(u)\right\|_{L^{2}\left(S^{1}\right)}^{2}+\left\|\mathscr{S}_{h}(v)-v\right\|_{L^{2}\left(S^{1}\right)}^{2}\right) .
\end{aligned}
$$

Thus, choosing $W=\mathscr{P}_{h}(u)$ and using approximation properties of the $L^{2}$ projector as well as the bound from Lemma 3.8 we have

$$
\left\|W-\mathscr{R}_{h}(u)\right\|_{V(h)}^{2} \leq C h_{8}^{2 q}\left(|u|_{H^{q+1}\left(S^{1}\right)}^{2}+\|v\|_{W^{q+1, \infty}\left(S^{1}\right)}^{2}\right),
$$


and hence the $V(h)$ norm bound follows from the triangle inequality and the definition of $v$. To show the $L^{2}$ bound, let $z \in H^{2}\left(S^{1}\right)$ solve the dual problem

$$
-z_{x x}+z=u-\mathscr{R}_{h}(u)
$$

then elliptic regularity guarantees that

$$
|z|_{H^{2}\left(S^{1}\right)} \leq C\left\|u-\mathscr{R}_{h}(u)\right\|_{L^{2}\left(S^{1}\right)} .
$$

Hence, for any $Z \in \mathbb{V}_{q}$

$$
\begin{aligned}
\left\|u-\mathscr{R}_{h}(u)\right\|_{L^{2}\left(S^{1}\right)}^{2} & =\int_{S^{1}}\left(u-\mathscr{R}_{h}(u)\right)^{2} \mathrm{~d} x \\
& =\int_{S^{1}}\left(-z_{x x}+z\right)\left(u-\mathscr{R}_{h}(u)\right) \mathrm{d} x \\
& =\mathscr{A}_{h}\left(z, u-\mathscr{R}_{h}(u)\right)+\int_{S^{1}} z\left(u-\mathscr{R}_{h}(u)\right) \mathrm{d} x \\
& =\mathscr{A}_{h}\left(z-Z, u-\mathscr{R}_{h}(u)\right)+\int_{S^{1}}(z-Z)\left(u-\mathscr{R}_{h}(u)\right)+\left(\mathscr{S}_{h}(v)-v\right) Z \mathrm{~d} x,
\end{aligned}
$$

by the quasi-orthogonality result (3.47). Making use of the orthogonality of $\mathscr{S}_{h}$, we choose $Z \in \mathbb{V}_{q-1}$ as the $L^{2}$ orthogonal projector of $z$ and find, by Cauchy-Schwarz

$$
\begin{aligned}
\left\|u-\mathscr{R}_{h}(u)\right\|_{L^{2}\left(S^{1}\right)}^{2} & \leq C_{b}\|z-Z\|_{V(h)}\left\|u-\mathscr{R}_{h}(u)\right\|_{V(h)}+\|z-Z\|_{L^{2}\left(S^{1}\right)}\left\|u-\mathscr{R}_{h}(u)\right\|_{L^{2}\left(S^{1}\right)} \\
& \leq C h|z|_{2}\left\|u-\mathscr{R}_{h}(u)\right\|_{V(h)}+C h^{2}|z|_{2}\left\|u-\mathscr{R}_{h}(u)\right\|_{L^{2}\left(S^{1}\right)} \\
& \leq C h\left\|u-\mathscr{R}_{h}(u)\right\|_{L^{2}\left(S^{1}\right)}\left\|u-\mathscr{R}_{h}(u)\right\|_{V(h)}+C h^{2}\left\|u-\mathscr{R}_{h}(u)\right\|_{L^{2}\left(S^{1}\right)}^{2}
\end{aligned}
$$

using the elliptic regularity result (3.53) and approximation properties of the $L^{2}$ projector. Hence

$$
\left(1-C h^{2}\right)\left\|u-\mathscr{R}_{h}(u)\right\|_{L^{2}\left(S^{1}\right)} \leq C h\left\|u-\mathscr{R}_{h}(u)\right\|_{V(h)}
$$

as required for $h$ small enough.

Theorem 3.12 (A priori bound - linear case). Suppose $f(u)=\frac{1}{2} u^{2}$, in this case the PDE (2.1) is linear and given by

$$
u_{t}-u_{x}+u_{x x x}=0 \text {. }
$$

Assume that the solution of (2.1) $u \in W^{q+4, \infty}\left(S^{1}\right)$ and $u_{t} \in W^{q+3, \infty}\left(S^{1}\right)$ and let $U$ solve (3.11) for some $q$ even and $N$ odd. Then, for $t \in[0, T]$ and $h$ small enough,

$$
\begin{aligned}
\frac{1}{2}\|(u-U)(t)\|_{V(h)}^{2}+\frac{1}{2}\|(u-U)(t)\|_{L^{2}\left(S^{1}\right)}^{2} \leq & \exp (t)\left(\frac{1}{2}\|(u-U)(0)\|_{V(h)}^{2}+\frac{1}{2}\|(u-U)(0)\|_{L^{2}\left(S^{1}\right)}^{2}\right. \\
& \left.+C h^{2 q} \int_{0}^{t}\left\|u_{t}\right\|_{W^{q+3, \infty}\left(S^{1}\right)}^{2}+\|u\|_{W^{q+4, \infty}\left(S^{1}\right)} \mathrm{d} s\right) .
\end{aligned}
$$

Proof. We begin by noting that, since $f^{\prime}(u)=u$, in Lemma 3.7 hence

$$
\frac{\mathrm{d}}{\mathrm{d} t} F_{1, h}\left(\theta^{u}\right)=\int_{S^{1}} \theta^{u} E^{u}-E^{v} \mathscr{G}_{h}\left(\theta^{v}\right) \mathrm{d} x+\mathscr{A}_{h}\left(\theta^{u}, E^{u}\right) .
$$

Observe that the term $\mathscr{G}_{h}\left(\theta^{v}\right)$ is not controllable in $F_{1, h}\left(\theta^{u}\right)$ and also will not be of an optimal order. It is prudent for fixed $(U, V)$ to choose $(\widetilde{U}, \widetilde{V})$ such that $E^{v}=0$. This then constrains choices for the pair $(\widetilde{U}, \widetilde{V})$. We pick $\widetilde{V}=\mathscr{S}_{h}(v)$ and then choose $\widetilde{U}=\mathscr{R}_{h}(u)$. This choice ensures that the perturbed equations

$$
\begin{aligned}
\int_{S^{1}} \widetilde{U}_{t} \Phi+\mathscr{G}_{h}(\widetilde{V}) \Phi \mathrm{d} x=\int_{S^{1}}-E^{u} \Phi \mathrm{d} x & \forall \Phi \in \mathbb{V}_{q} \\
\int_{S^{1}} \widetilde{V} \Psi+f^{\prime}(\widetilde{U}) \Psi \mathrm{d} x+\mathscr{A}_{h}(\widetilde{U}, \Psi)=\int_{S^{1}}-E^{v} \Psi \mathrm{d} x & \forall \Psi \in \mathbb{V}_{q},
\end{aligned}
$$

are satisfied with

$$
\begin{aligned}
E^{u} & =u_{t}-\widetilde{U}_{t}+v_{x}-\mathscr{G}_{h}(\widetilde{V}) \\
E^{v} & =0 .
\end{aligned}
$$

Substituting this into (3.59) we have

$$
\frac{\mathrm{d}}{\mathrm{d} t} F_{1, h}\left(\theta^{u}\right)=\int_{S^{1}} \theta^{u} E^{u} \mathrm{~d} x+\mathscr{A}_{h}\left(\theta^{u}, E^{u}\right)
$$


Now, through Cauchy's inequality we see

$$
\frac{\mathrm{d}}{\mathrm{d} t} F_{1, h}\left(\theta^{u}\right) \leq \frac{1}{2}\left(\left\|\theta^{u}\right\|_{L^{2}\left(S^{1}\right)}^{2}+C_{c}\left\|\theta^{u}\right\|_{V(h)}^{2}+\left\|E^{u}\right\|_{L^{2}\left(S^{1}\right)}^{2}+\frac{C_{b}^{2}}{C_{c}}\left\|E^{u}\right\|_{V(h)}^{2}\right) .
$$

Now since

$$
\frac{C_{c}}{2}\left\|\theta^{u}\right\|_{V(h)}^{2}+\frac{1}{2}\left\|\theta^{u}\right\|_{L^{2}\left(S^{1}\right)}^{2} \leq F_{1, h}\left(\theta^{u}\right),
$$

Gronwall's inequality implies

$$
\begin{aligned}
C_{c}\left\|\theta^{u}(t)\right\|_{V(h)}^{2}+\left\|\theta^{u}(t)\right\|_{L^{2}\left(S^{1}\right)}^{2} \leq \exp (t) & \left(C_{c}\left\|\theta^{u}(0)\right\|_{V(h)}^{2}+\left\|\theta^{u}(0)\right\|_{L^{2}\left(S^{1}\right)}^{2}\right. \\
& \left.+\int_{0}^{t}\left\|E^{u}(s)\right\|_{L^{2}\left(S^{1}\right)}^{2}+\frac{C_{b}^{2}}{C_{c}}\left\|E^{u}(s)\right\|_{V(h)}^{2} \mathrm{~d} s\right) .
\end{aligned}
$$

It remains to bound the term $E^{u}$. We do this by splitting into two components and controlling them individually. First note that since we are in a semi discrete setting, Lemma 3.11 yields

$$
\left\|u_{t}-\mathscr{R}_{h}\left(u_{t}\right)\right\|_{L^{2}\left(S^{1}\right)} \leq C h^{q+1}\left\|u_{t}\right\|_{W^{q+3, \infty}\left(S^{1}\right)} .
$$

Further, Lemma 3.8 immediately gives

$$
\left\|v_{x}-\mathscr{G}_{h}\left(\mathscr{S}_{h}(v)\right)\right\|_{L^{2}\left(S^{1}\right)} \leq C h^{q+1}\|u\|_{W^{q+4, \infty}\left(S^{1}\right)},
$$

hence

$$
\left\|E^{u}\right\|_{L^{2}\left(S^{1}\right)}^{2}+C_{b}^{2}\left\|E^{u}\right\|_{V(h)}^{2} \leq C h^{2 q}\left(\left\|u_{t}\right\|_{W^{q+3, \infty}\left(S^{1}\right)}^{2}+\|u\|_{W^{q+4, \infty}\left(S^{1}\right)}^{2}\right),
$$

as required.

Remark 3.13 (A priori bound - nonlinear case). In the nonlinear case, for $f(u)=\alpha u^{m}$, for $m>2$, our problem is given by

$$
u_{t}-\alpha\left(u^{m}\right)_{x}+u_{x x x}=0 .
$$

An optimal bound for this using the methodology proposed above requires the appropriate handling of discontinuous Galerkin approximations of the associated Emden-Fowler type elliptic problem

$$
-u_{x x}+|u|^{m-2} u=f
$$

which is discussed in [28]. It should be noted that optimal a priori control of approximations to this problem even in the energy norm are not trivial for $m>2$.

\section{A posteriori ANALysis}

In this section, we give an a posteriori analysis of the semi discrete scheme posed in Section 3 We proceed along similar lines to the a priori analysis in that we examine solutions of perturbed equations, taking account of different effects errors induced will have. The difference being, in this section we make use of the stability framework of the underlying PDE.

Lemma 4.1. Let $u \in C^{1}\left([0, T], H^{3}\left(S^{1}\right)\right)$ be a strong solution to (2.1) and suppose $\widetilde{u} \in C^{1}\left([0, T], H^{3}\left(S^{1}\right)\right)$ satisfies the problem

$$
\begin{aligned}
\widetilde{u}_{t}-f^{\prime}(u)_{x}+f^{\prime}(u-\widetilde{u})_{x}+\widetilde{u}_{x x x} & =-\mathfrak{E}, \\
\widetilde{u}(x, 0) & =\widetilde{u}^{0}(x)
\end{aligned}
$$

for some $\mathfrak{E} \in L^{2}\left(S^{1}\right)$. Then, with $\rho:=u-\widetilde{u}$

$$
\frac{\mathrm{d}}{\mathrm{d} t}\left(\int_{S^{1}} \frac{1}{2} \rho_{x}^{2}+f(\rho) \mathrm{d} x\right)=\int_{S^{1}}\left(-\rho_{x x}+f^{\prime}(\rho)\right) \mathfrak{E} \mathrm{d} x
$$

Proof. To begin, we note that $\rho=u-\widetilde{u}$ satisfies the error equation

$$
\rho_{t}-f^{\prime}(\rho)_{x}+\rho_{x x x}=\mathfrak{E} .
$$

Then, explicitly computing the time derivative we have

$$
\begin{aligned}
\frac{\mathrm{d}}{\mathrm{d} t} F_{1}(\rho) & =\int_{S^{1}} \rho_{x} \rho_{x t}+f^{\prime}(\rho) \rho_{t} \mathrm{~d} x \\
& =\int_{S^{1}}-\rho_{x x} \rho_{t}+f^{\prime}(\rho) \rho_{t} \mathrm{~d} x .
\end{aligned}
$$


Making use of (4.3) we see

$$
\begin{aligned}
\int_{S^{1}}-\rho_{x x} \rho_{t}+f^{\prime}(\rho) \rho_{t} \mathrm{~d} x & =\int_{S^{1}}\left(-\rho_{x x}+f^{\prime}(\rho)\right)\left(\mathfrak{E}-\rho_{x x x}+f^{\prime}(\rho)_{x}\right) \mathrm{d} x \\
& =\int_{S^{1}}\left(-\rho_{x x}+f^{\prime}(\rho)\right)\left(\left(-\rho_{x x}+f^{\prime}(\rho)\right)_{x}+\mathfrak{E} \mathrm{d} x\right) \\
& =\int_{S^{1}}\left(-\rho_{x x}+f^{\prime}(\rho)\right) \mathfrak{E} \mathrm{d} x,
\end{aligned}
$$

as required.

Definition 4.2 (Orthogonal decomposition). To make use of the stability framework of the PDE, we split the numerical solution, $U \in \mathbb{V}_{q}$ into a continuous component and a discontinuous component

$$
U=U^{c}+U^{d},
$$

where $U^{c} \in C^{1}\left(0, T ; \mathbb{V}_{q} \cap C^{0}\left(S^{1}\right)\right)$ and $U^{d}:=U-U^{c} \in C^{1}\left(0, T ; \mathbb{V}_{q}\right)$. We decompose in such a way that

$$
\mathscr{A}_{h}\left(U^{d}, \Phi\right)=0 \quad \forall \Phi \in \mathbb{V}_{q} \cap C^{0}\left(S^{1}\right),
$$

as in [14]. We will make use of the following result, the proof of which can be found in [26].

Proposition 4.3 (Bound on nonconforming term). Let $U=U^{c}+U^{d}$ be the orthogonal decomposition from Definition 4.2 then we have

$$
\left\|U^{d}\right\|_{V(h)}^{2} \leq C \sum_{j=0}^{N-1}\{h\}_{j}^{-1} \llbracket U \rrbracket_{j}^{2},
$$

where $C$ depends only upon $q$ and the $\max _{j} \frac{h_{j}}{h_{j+1}}$.

Theorem 4.4 (Discrete reconstruction operator $\mathscr{D}_{h}$ ). For each $W \in \mathbb{V}_{q}$ there exists a unique $\mathscr{D}_{h}(W) \in \mathbb{V}_{q+1} \cap C^{0}\left(S^{1}\right)$ such that for $j=0, \ldots, N-1$

$$
\begin{aligned}
\int_{S^{1}} \mathscr{D}_{h}(W)_{x} \Phi \mathrm{d} x & =\int_{S^{1}} \mathscr{G}_{h}(W) \Phi \mathrm{d} x \quad \forall \Phi \in \mathbb{V}_{q}, \quad t>0 \\
\mathscr{D}_{h}(W)\left(x_{j}^{+}\right) & =\{W\}_{j} \quad \forall j=0, \ldots N-1 .
\end{aligned}
$$

Moreover, $\mathscr{D}_{h}$ satisfies

$$
\left\|W-\mathscr{D}_{h}(W)\right\|_{L^{2}\left(S^{1}\right)}^{2} \leq C \sum_{j=0}^{N-1}\{h\}_{j} \llbracket W \rrbracket_{j}^{2} .
$$

Proof. Fix $W \in \mathbb{V}_{q}$, then a candidate $\Psi \in \mathbb{V}_{q}$ given by

$$
\int_{x_{j}}^{x_{j+1}} \Psi \Phi \mathrm{d} x=\int_{x_{j}}^{x_{j+1}} \mathscr{G}_{h}(W) \Phi \mathrm{d} x \quad \forall j=0, \ldots N-1
$$

exists through Riesz Representation Theorem. Now, with $\mathscr{D}_{h}(W) \in \mathbb{V}_{q+1}$ as the anti spatial derivative of $\Psi$, the constant of integration can be chosen such that the boundary condition is satisfied showing existence and uniqueness.

To show $\mathscr{D}_{h}(W) \in C^{0}\left(S^{1}\right)$ we fix $j$ and consider

$$
\Phi(x)=\left\{\begin{array}{l}
1 \text { if } x \in\left(x_{j}, x_{j+1}\right) \\
0 \text { otherwise }
\end{array}\right.
$$

Now note that by definition, and the periodic boundary conditions, we have

$$
\begin{aligned}
0 & =\int_{x_{j}}^{x_{j+1}} \mathscr{D}_{h}(W)_{x} \Phi-\mathscr{G}_{h}(W) \Phi \mathrm{d} x \\
& =\mathscr{D}_{h}(W)\left(x_{j+1}^{-}\right)-\mathscr{D}_{h}(W)\left(x_{j}^{+}\right)-\{W\}_{j+1}+\{W\}_{j} \\
& =\mathscr{D}_{h}(W)\left(x_{j+1}^{-}\right)-\{W\}_{j+1} .
\end{aligned}
$$

This ensures $\mathscr{D}_{h}(W)$ is continuous over the edge and, since $j$ was arbitrary, globally. To show the approximation properties we note that $\mathscr{D}_{h}(W)-W \in \mathbb{V}_{q+1}$ and, in particular,

$$
\int_{x_{j}}^{x_{j+1}}\left(\mathscr{D}_{h}(W)-W\right) \Phi \mathrm{d} x=0 \quad \forall \Phi \in \mathbb{V}_{q-1} .
$$


We can then write the difference in terms of the Legendre polynomials defined in the Proof of Lemma 3.8. In particular, due to the orthogonality condition (4.14) we have

$$
\left.\left(\mathscr{D}_{h}(W)-W\right)\right|_{I_{j}}=\sum_{k=0}^{q+1} \alpha_{j, k} l_{j, k}(x)=\alpha_{j, q} l_{j, q}(x)+\alpha_{j, q+1} l_{j, q+1}(x) \text { for } j=0, \ldots, N-1
$$

and endpoint conditions

$$
\begin{aligned}
\left(\mathscr{D}_{h}(W)-W\right)\left(x_{j}^{+}\right) & =\{W\}_{j}-W_{j}^{+}=\frac{1}{2} \llbracket W \rrbracket_{j} \\
\left(\mathscr{D}_{h}(W)-W\right)\left(x_{j+1}^{-}\right) & =\{W\}_{j+1}-W_{j+1}^{-}=-\frac{1}{2} \llbracket W \rrbracket_{j+1} .
\end{aligned}
$$

Making use of the properties of the Legendre polynomials, we can write the linear system

$$
\begin{aligned}
\alpha_{j, q}(-1)^{q}+\alpha_{j, q+1}(-1)^{q+1} & =\frac{1}{2} \llbracket W \rrbracket_{j} \\
\alpha_{j, q}+\alpha_{j, q+1} & =-\frac{1}{2} \llbracket W \rrbracket_{j} .
\end{aligned}
$$

This can then be readily solved to show

$$
\begin{aligned}
\alpha_{j, q} & =\frac{1}{4}\left((-1)^{q} \llbracket W \rrbracket_{j}-\llbracket W \rrbracket_{j+1}\right) \\
\alpha_{j, q+1} & =\frac{1}{4}\left((-1)^{q+1} \llbracket W \rrbracket_{j}-\llbracket W \rrbracket_{j+1}\right),
\end{aligned}
$$

giving an explicit representation for $\left.\left(\mathscr{D}_{h}(W)-W\right)\right|_{I_{j}}$. Hence

$$
\begin{aligned}
\int_{x_{j}}^{x_{j+1}}\left(\mathscr{D}_{h}(W)-W\right)^{2} \mathrm{~d} x & =\int_{x_{j}}^{x_{j+1}} \alpha_{j, q}^{2} l_{j, q}(x)^{2}+\alpha_{j, q+1}^{2} l_{j, q+1}(x)^{2} \mathrm{~d} x \\
& =\alpha_{j, q}^{2} \frac{h_{j}}{2 q+1}+\alpha_{j, q+1}^{2} \frac{h_{j}}{2 q+3} \\
& \leq \frac{h_{j}}{8(2 q+1)}\left(\llbracket W \rrbracket_{j}^{2}+\llbracket W \rrbracket_{j+1}^{2}\right) .
\end{aligned}
$$

The result follows by summing over all elements with $C=(4(2 q+1))^{-1}$ if the mesh is uniform. In the nonuniform setting $C$ depends on $\max _{j} \frac{h_{j}}{h_{j+1}}$, the grading of the mesh.

Definition 4.5 (Elliptic reconstruction). Let $(U, V) \in \mathbb{V}_{q} \times \mathbb{V}_{q}$ be the semi-discrete approximation given by (3.11). Then, the elliptic reconstruction $\omega(U): \mathbb{V}_{q} \rightarrow H^{3}\left(S^{1}\right)$ is given by the solution of

$$
-\omega(U)_{x x}+f^{\prime}(\omega(U))=\mathscr{D}_{h}(V)
$$

with average value matching the discrete solution, that is

$$
\int_{S^{1}} \omega(U)-U \mathrm{~d} x=0
$$

Remark 4.6 (Inconsistent elliptic reconstruction). The reconstruction $\omega(U)$ is an inconsistent elliptic reconstruction of $U$ [37]. Indeed, $U$ is the finite element approximation of

$$
-\omega(U)_{x x}+f^{\prime}(\omega(U))=V .
$$

The reason for defining $\omega(U)$ as in Definition 4.5 is to ensure that the reconstruction has sufficient regularity to satisfy the perturbed PDE in Lemma 4.1.

Proposition 4.7 (Regularity bound for the reconstruction). The elliptic problem defining the reconstruction operator, $\omega(U)$, in Definition 4.5 is well posed, moreover, thanks to elliptic regularity, we have

$$
\|\omega(U)\|_{H^{k+1}\left(S^{1}\right)} \leq C_{\text {reg }}\left\|\mathscr{D}_{h}(V)\right\|_{H^{k-1}\left(S^{1}\right)} \quad \text { for } k=0,1,2 .
$$

Lemma 4.8 (Reconstructed PDE). The reconstruction given in Definition 4.5 satisfies

$$
\omega(U)_{t}-f^{\prime}(u)_{x}+f^{\prime}(u-\omega(U))_{x}+\omega(U)_{x x x}=\mathfrak{E},
$$

with

$$
\mathfrak{E}=(\omega(U)-U)_{t}+f^{\prime}(\omega(U))_{x}-f^{\prime}(u)_{x}+f^{\prime}(u-\omega(U))_{x} .
$$


Proof. Since $\omega(U)$ satisfies (4.20) and the problem data $\mathscr{D}_{h}(V) \in H^{1}\left(S^{1}\right)$ it is clear that $\omega(U) \in H^{3}\left(S^{1}\right)$ and satisfies

$$
-\omega(U)_{x x x}+f^{\prime}(\omega(U))_{x}=\mathscr{D}_{h}(V)_{x}=U_{t},
$$

upon writing the definition of $\mathscr{D}_{h}(V)$ point-wise. Now, substituting (4.26) into (4.24), we see

$$
\omega(U)_{t}-f^{\prime}(u)_{x}+f^{\prime}(u-\omega(U))_{x}+\omega(U)_{x x x}=(\omega(U)-U)_{t}+f^{\prime}(\omega(U))_{x}-f^{\prime}(u)_{x}+f^{\prime}(u-\omega(U))_{x},
$$

as required.

Hypothesis 4.9 (A posteriori control for the elliptic problem). We make the assumption that there exists an optimal order elliptic a posteriori estimate controlling the energy norm error. That is, there exists a functional $\eta$ depending only upon $U$ and the problem data such that

$$
\left(\|U-\omega(U)\|_{L^{m}\left(S^{1}\right)}^{m}+\|U-\omega(U)\|_{\mathbb{V}_{q}}^{2}\right)^{1 / 2} \leq \eta\left(U, g, H^{1}\left(S^{1}\right)\right) .
$$

Example 4.10. For $f(u)=\frac{1}{m} u^{m}$ with $g:=-\mathscr{D}_{h}(V)$ in [28] it was shown that

$$
\begin{aligned}
\|U-\omega(U)\|_{L^{m}\left(S^{1}\right)}^{m}+\|U-\omega(U)\|_{\mathbb{V}_{q}}^{2} \leq & C \sum_{j=0}^{N-1}\left[\left\|h\left(g+U_{x x}-f^{\prime}(U)\right)\right\|_{L^{2}\left(x_{j}, x_{j+1}\right)}^{2}\right. \\
& \left.+\{h\}_{j} \llbracket U_{x} \rrbracket_{j}^{2}+\sigma\{h\}_{j}^{-1} \llbracket U \rrbracket_{j}^{2}+\{h\}_{j} \llbracket V \rrbracket_{j}^{2}\right]
\end{aligned}
$$

satisfies Hypothesis 4.9 .

Note that the inconsistency described in Remark 4.6 induced by modifying the elliptic reconstruction is accounted for by the last term in this estimator.

Remark 4.11 (Alternative estimators). One of the strengths of the elliptic reconstruction methodology is the ability to use estimators that are not residual based. Indeed, recovery based a posteriori estimators have been widely used since their introduction by the engineering community in the 1980s. Their success is due to their simplicity of implementation, mild dependence of problem data and super-convergence properties. Work carried out on recovery estimators has reached a state of maturity for elliptic problems, see [2, 6, 34, 49] and subsequent references. These estimators could also be used in the subsequent analysis.

Theorem 4.12 (A posteriori bound - linear case). Suppose $f(u)=\frac{1}{2} u^{2}$. Further, let $U$ solve (3.11) and $\omega(U)$ be the elliptic reconstruction from Definition 4.5. Let the conditions of Lemma 4.1 hold. Then, with $\rho:=u-\omega(U)$, for $t \in[0, T]$,

$$
\begin{aligned}
F_{1}(\rho(t)) \leq \exp (t)\left(F_{1}(\rho(0))\right. & +\int_{0}^{t}\left\|\left(\omega(U)_{t}-U_{t}\right)(s)\right\|_{L^{2}\left(S^{1}\right)}^{2} \\
& \left.+2\left\|\left(\omega(U)_{t}-U_{t}^{c}\right)_{x}(s)\right\|_{L^{2}\left(S^{1}\right)}^{2}+2 C_{a}^{2} C_{b}^{2}\left\|U_{t}^{d}(s)\right\|_{V(h)}^{2} \mathrm{~d} s\right) .
\end{aligned}
$$

Proof. Since $f^{\prime}(u)=u$, in Lemma 4.1 $\mathfrak{E}=\omega(U)_{t}-U_{t}$, hence

$$
\begin{aligned}
\mathrm{d}_{t} F_{1}(\rho) & =\int_{S^{1}}\left(-\rho_{x x}+\rho\right) \mathfrak{E} \mathrm{d} x \\
& =\mathscr{A}_{h}\left(\rho, \omega(U)_{t}-U_{t}\right)+\rho\left(\omega(U)_{t}-U_{t}\right) \mathrm{d} x .
\end{aligned}
$$

Now making use of the orthogonal decomposition of $U=U^{c}+U^{d}$ given in (4.7) we have

$$
\begin{aligned}
\mathscr{A}_{h}\left(\rho, \omega(U)_{t}-U_{t}\right) & =\int_{S^{1}} \rho_{x}\left(\omega(U)_{t}-U_{t}^{c}\right)_{x} \mathrm{~d} x-\mathscr{A}_{h}\left(\rho, U_{t}^{d}\right) \\
& =\int_{S^{1}} \rho_{x}\left(\omega(U)_{t}-U_{t}^{c}\right)_{x} \mathrm{~d} x-\mathscr{A}_{h}\left(\rho-P, U_{t}^{d}\right) \quad \forall P \in \mathbb{V}_{q} \cap H^{1}\left(S^{1}\right),
\end{aligned}
$$

since $\llbracket P \rrbracket=0$. Choosing $P=I_{h}(\rho)$ as the Scott-Zhang interpolant of $\rho$, we have

$$
\mathscr{A}_{h}\left(\rho, \omega(U)_{t}-U_{t}\right) \leq \frac{1}{4}\left\|\rho_{x}\right\|_{L^{2}\left(S^{1}\right)}^{2}+\left\|\left(\omega(U)_{t}-U_{t}^{c}\right)_{x}\right\|_{L^{2}\left(S^{1}\right)}^{2}+\epsilon\left\|\rho-I_{h}(\rho)\right\|_{V(h)}^{2}+\frac{C_{b}^{2}}{4 \epsilon}\left\|U_{t}^{d}\right\|_{V(h)}^{2} .
$$

Using the stability of the Scott-Zhang interpolant in mesh dependent norms, see [17, Lemma 3.49 c.f.] we have

$$
\left\|\rho-I_{h}(\rho)\right\|_{V(h)} \leq C_{a}\left\|\rho_{x}\right\|_{L^{2}\left(S^{1}\right)} .
$$

Hence, for any $\epsilon>0$

$$
\begin{aligned}
\mathscr{A}_{h}\left(\rho, \omega(U)_{t}-U_{t}\right) & \leq \frac{1}{4}\left\|\rho_{x}\right\|_{L^{2}\left(S^{1}\right)}^{2}+\left\|\left(\omega(U)_{t}-U_{t}^{c}\right)_{x}\right\|_{L^{2}\left(S^{1}\right)}^{2}+C_{a}^{2} \epsilon\left\|\rho_{x}\right\|_{L^{2}\left(S^{1}\right)}^{2}+\frac{C_{b}^{2}}{4 \epsilon}\left\|U_{t}^{d}\right\|_{V(h)}^{2} \\
& \leq \frac{1}{2}\left\|\rho_{x}\right\|_{L^{2}\left(S^{1}\right)}^{2}+\left\|\left(\omega(U)_{t}-U_{t}^{c}\right)_{x}\right\|_{L^{2}\left(S^{1}\right)}^{2}+C_{a}^{2} C_{b}^{2}\left\|U_{t}^{d}\right\|_{V(h)}^{2}
\end{aligned}
$$


with $\epsilon=1 /\left(4 C_{a}^{2}\right)$. Substituting (4.35) into (4.31) we have

$$
\frac{\mathrm{d}}{\mathrm{d} t} F_{1}(\rho) \leq \frac{1}{2}\left\|\rho_{x}\right\|_{L^{2}\left(S^{1}\right)}^{2}+\left\|\left(\omega(U)_{t}-U_{t}^{c}\right)_{x}\right\|_{L^{2}\left(S^{1}\right)}^{2}+\frac{1}{2}\|\rho\|_{L^{2}\left(S^{1}\right)}^{2}+\frac{1}{2}\left\|\omega(U)_{t}-U_{t}\right\|_{L^{2}\left(S^{1}\right)}^{2}+C_{a}^{2} C_{b}^{2}\left\|U_{t}^{d}\right\|_{V(h)}^{2} .
$$

The result follows from Gronwall's inequality.

Corollary 4.13 (Computable a posteriori bound - linear case). Let the conditions of Theorem 4.12 hold. Then, with $e:=u-U$, for $t \in[0, T]$,

$$
\begin{aligned}
& \frac{1}{2}\|e(t)\|_{\mathbb{V}_{q}}^{2}+\frac{1}{2}\|e(t)\|_{L^{2}\left(S^{1}\right)}^{2} \leq \eta\left(U(t), g, H^{1}\left(S^{1}\right)\right)^{2} \\
& +2 \exp (t)\left(F_{1}(\rho(0))+\int_{0}^{t} 2 \eta\left(U_{s}(s), g_{s}, H^{1}\left(S^{1}\right)\right)^{2}\right. \\
& \left.+\left(2 C_{a}^{2} C_{b}^{2}\right)\left\|U_{s}^{d}(s)\right\|_{V(h)}^{2} \mathrm{~d} s\right) \\
& =: E_{s t}(U(t)) .
\end{aligned}
$$

Note that $E_{\text {st }}$ is the computed quantity in the numerical results in Section 6 .

Proof. We begin by noting

$$
\frac{1}{4}\|e\|_{\mathbb{V}_{q}}^{2}+\frac{1}{4}\|e\|_{L^{2}\left(S^{1}\right)}^{2} \leq F_{1}(\rho)+\frac{1}{2}\|\theta\|_{\mathbb{V}_{q}}^{2}+\frac{1}{2}\|\theta\|_{L^{2}\left(S^{1}\right)}^{2},
$$

where $\rho:=u-\omega(U)$ and $\theta:=\omega(U)-U$. Invoking Hypothesis 4.9 and applying Theorem 4.12 we may conclude.

Remark 4.14 (The linear vs nonlinear case). Notice that when $f(u)=\frac{1}{2} u^{2}$ we have that $\widetilde{u}$ solves a compatible KdV-like problem

$$
\widetilde{u}_{t}-\widetilde{u}_{x}+\widetilde{u}_{x x x}=-\mathfrak{E} .
$$

This makes the analysis considerably simpler than for general $f$. For expositions sake in the rest of this section we will only consider the case $f(u)=\frac{1}{4} u^{4}$, corresponding to the defocusing $m K d V$ equation. The arguments for more general non-linearity are lengthy and we wish to highlight that our analysis allows for explicit control on the constants appearing in the a posteriori upper bound, something very challenging for nonlinear evolution problems.

Lemma 4.15 (A priori solution control). Let $u$ solve (2.1) and $\omega(U)$ be the elliptic reconstruction given in Definition 4.5. Then, with $f(u)=\frac{1}{4} u^{4}$, and for an initial condition $u^{0} \in H^{2}\left(S^{1}\right)$, we have the following

$$
\left\|(u \omega(U))_{x}\right\|_{L^{\infty}\left(S^{1}\right)} \leq\left\|(u \omega(U))_{x x}\right\|_{L^{2}\left(S^{1}\right)} \leq C_{4},
$$

with

$$
\begin{aligned}
C_{4}:= & C_{r e g}\left(\left\|\mathscr{D}_{h}(V)\right\|_{H^{-1}\left(S^{1}\right)}\left(\left\|u_{x x}^{0}\right\|_{L^{2}\left(S^{1}\right)}^{2}+5\left\|u^{0} u_{x}^{0}\right\|_{L^{2}\left(S^{1}\right)}^{2}+\frac{1}{2}\left\|u^{0}\right\|_{L^{6}\left(S^{1}\right)}^{6}\right)^{1 / 2}\right. \\
& \left.+\left\|\mathscr{D}_{h}(V)\right\|_{L^{2}\left(S^{1}\right)}\left(\left\|u_{x}^{0}\right\|_{L^{2}\left(S^{1}\right)}^{2}+\left\|u^{0}\right\|_{L^{4}\left(S^{1}\right)}^{4}\right)^{1 / 2}\right) .
\end{aligned}
$$

Proof. We begin by noting that with (2.1) admits a further invariant. That is,

$$
\frac{\mathrm{d}}{\mathrm{d} t} \int_{S^{1}} 2 u_{x x}^{2}+10 u^{2} u_{x}^{2}+u^{6} \mathrm{~d} x=0 .
$$

Hence we have

$$
\left\|u_{x x}\right\|_{L^{2}\left(S^{1}\right)} \leq\left(\left\|u_{x x}^{0}\right\|_{L^{2}\left(S^{1}\right)}^{2}+5\left\|u^{0} u_{x}^{0}\right\|_{L^{2}\left(S^{1}\right)}^{2}+\frac{1}{2}\left\|u^{0}\right\|_{L^{6}\left(S^{1}\right)}^{6}\right)^{1 / 2} .
$$

In addition, since

$$
\frac{\mathrm{d}}{\mathrm{d} t} \int_{S^{1}} \frac{1}{2} u_{x}^{2}+\frac{1}{4} u^{4} \mathrm{~d} x=0
$$

we have

$$
\left\|u_{x}\right\|_{L^{2}\left(S^{1}\right)} \leq\left(\left\|u_{x}^{0}\right\|_{L^{2}\left(S^{1}\right)}^{2}+\frac{1}{2}\left\|u^{0}\right\|_{L^{4}\left(S^{1}\right)}^{4}\right)^{1 / 2}
$$

The result (4.40) follows from (4.43), (4.45) and Proposition 4.7 and an interpolation argument. 
Theorem 4.16 (A posteriori bound - nonlinear case $m=4$ ). Suppose $f(u)=\frac{1}{4} u^{4}$. Further, let $U$ solve (3.11) and the conditions of Lemmas 4.1 and 4.8 hold. Then, with $\rho:=u-\omega(U)$, for $t \in[0, T]$,

$$
\begin{aligned}
F_{1}(\rho(t)) \leq \exp \left(\mathscr{C}_{4} t\right)\left(F_{1}(\rho(0))+\int_{0}^{t}\right. & \frac{1}{4}\left\|\left(\omega(U)_{t}-U_{t}\right)(s)\right\|_{L^{m}\left(S^{1}\right)}^{4} \\
& \left.+\left\|\left(\omega(U)_{t}-U_{t}^{c}\right)_{x}(s)\right\|_{L^{2}\left(S^{1}\right)}^{2}+C_{a}^{2} C_{b}^{2}\left\|U_{t}^{d}(s)\right\|_{V(h)}^{2} \mathrm{~d} s\right),
\end{aligned}
$$

with

$$
\mathscr{C}_{4}=\max \left(\frac{1}{2}+\frac{15}{2} C_{4}, \frac{3}{4}+\frac{9}{4} C_{4}\right)
$$

Proof. For $m=4$, since $f^{\prime}(u)=u^{3}$ we have

$$
\begin{aligned}
\mathfrak{E} & =\omega(U)_{t}-U_{t}+f^{\prime}(\omega(U))_{x}-f^{\prime}(u)_{x}+f^{\prime}(u-\omega(U))_{x} \\
& =\omega(U)_{t}-U_{t}-(3 u \omega(U)(u-\omega(U)))_{x} .
\end{aligned}
$$

Initial inspection of the form of $\mathfrak{E}$ indicates the resultant bound should not be optimal. Surprisingly, this is not the case as the extra derivative can be "hidden" by requiring regularity of $u$ and $\omega(U)$, which we have already quantified in a computational fashion.

Through Lemma 4.1

$$
\begin{aligned}
\mathrm{d}_{t} F_{1}(\rho) & =\int_{S^{1}}\left(-\rho_{x x}+\rho^{3}\right) \mathfrak{E} \mathrm{d} x \\
& =\underbrace{\mathscr{A}_{h}\left(\rho, \omega(U)_{t}-U_{t}\right)}_{\mathscr{I}_{1}}-\underbrace{3 \mathscr{A}\left(\rho,(u \omega(U) \rho)_{x}\right)}_{\mathscr{I}_{2}}+\int_{S^{1}} \underbrace{\rho^{3}\left(\omega(U)_{t}-U_{t}\right)}_{\mathscr{I}_{3}}-\underbrace{3 \rho^{3}(u \omega(U) \rho)_{x}}_{\mathscr{I}_{4}} \mathrm{~d} x .
\end{aligned}
$$

We proceed to control these terms individually. To begin, arguing as in (4.33), making use of the orthogonal decomposition (4.7) with $P=I_{h}(\rho)$ as the Scott-Zhang interpolant of $\rho$

$$
\begin{aligned}
\mathscr{I}_{1} & =\mathscr{A}_{h}\left(\rho, \omega(U)_{t}-U_{t}\right) \\
& =\int_{S^{1}} \rho_{x}\left(\omega(U)_{t}-U_{t}^{c}\right)_{x} \mathrm{~d} x-\mathscr{A}_{h}\left(\rho-P, U_{t}^{d}\right) \\
& \leq \frac{1}{2}\left\|\rho_{x}\right\|_{L^{2}\left(S^{1}\right)}^{2}+\left\|\left(\omega(U)_{t}-U_{t}^{c}\right)_{x}\right\|_{L^{2}\left(S^{1}\right)}^{2}+C_{a}^{2} C_{b}^{2}\left\|U_{t}^{d}\right\|_{V(h)}^{2} .
\end{aligned}
$$

Now, through expanding derivatives

$$
3(u \omega(U) \rho)_{x x}=3(u \omega(U))_{x x} \rho+6(u \omega(U))_{x} \rho_{x}+3 u \omega(U) \rho_{x x},
$$

and integrating by parts we see

$$
\begin{aligned}
\mathscr{I}_{2} & =\int_{S^{1}} 3 \rho_{x}(u \omega(U) \rho)_{x x} \mathrm{~d} x \\
& =\int_{S^{1}} 3(u \omega(U))_{x x} \rho \rho_{x}+6(u \omega(U))_{x} \rho_{x}^{2}+3 u \omega(U) \rho_{x x} \rho_{x} \mathrm{~d} x .
\end{aligned}
$$

Now note that

$$
\begin{aligned}
\int_{S^{1}} 3 u \omega(U) \rho_{x x} \rho_{x} \mathrm{~d} x & =-\int_{S^{1}} 3\left(u \omega(U) \rho_{x}\right)_{x} \rho_{x} \mathrm{~d} x \\
& =-3 \int_{S^{1}}(u \omega(U))_{x} \rho_{x}^{2}+u \omega(U) \rho_{x x} \rho_{x} \mathrm{~d} x
\end{aligned}
$$

Hence

$$
\int_{S^{1}} 3 u \omega(U) \rho_{x x} \rho_{x} \mathrm{~d} x=-\frac{3}{2} \int_{S^{1}}(u \omega(U))_{x} \rho_{x}^{2}
$$

Substituting (4.54) into (4.52)

$$
\mathscr{I}_{2}=\int_{S^{1}} 3(u \omega(U))_{x x} \rho \rho_{x}+\frac{9}{2}(u \omega(U))_{x} \rho_{x}^{2} \mathrm{~d} x .
$$


Making use of Hölder's inequality and a Sobolev embedding

$$
\begin{aligned}
\mathscr{I}_{2} & \leq 3\left\|(u \omega(U))_{x x}\right\|_{L^{2}\left(S^{1}\right)}\left\|\rho_{x}\right\|_{L^{2}\left(S^{1}\right)}\|\rho\|_{L^{\infty}\left(S^{1}\right)}+\frac{9}{2}\left\|(u \omega(U))_{x}\right\|_{L^{\infty}\left(S^{1}\right)}\left\|\rho_{x}\right\|_{L^{2}\left(S^{1}\right)}^{2} \\
& \leq 3 C_{4}\left\|\rho_{x}\right\|_{L^{2}\left(S^{1}\right)}^{2}+\frac{9}{2} C_{4}\left\|\rho_{x}\right\|_{L^{2}\left(S^{1}\right)}^{2} \\
& \leq \frac{15}{2} C_{4}\left\|\rho_{x}\right\|_{L^{2}\left(S^{1}\right)}^{2}
\end{aligned}
$$

by Lemma 4.15. The third term

$$
\begin{aligned}
\mathscr{I}_{3} & =\int_{S^{1}} \rho^{3}\left(\omega(U)_{t}-U_{t}\right) \mathrm{d} x \\
& \leq\left\|\rho^{3}\right\|_{L^{\frac{4}{3}\left(S^{1}\right)}}\left\|\omega(U)_{t}-U_{t}\right\|_{L^{4}\left(S^{1}\right)} \\
& \leq\|\rho\|_{L^{4}\left(S^{1}\right)}^{3}\left\|\omega(U)_{t}-U_{t}\right\|_{L^{4}\left(S^{1}\right)} \\
& \leq \frac{3}{4}\|\rho\|_{L^{4}\left(S^{1}\right)}^{4}+\frac{1}{4}\left\|\omega(U)_{t}-U_{t}\right\|_{L^{4}\left(S^{1}\right)}^{4} .
\end{aligned}
$$

The final term can be controlled by noticing

$$
\mathscr{I}_{4}=3 \int_{S^{1}} \rho^{3}(u \omega(U) \rho)_{x} \mathrm{~d} x=3 \int_{S^{1}} \frac{1}{4}\left(\rho^{4}\right)_{x} u \omega(U)+\rho^{4}(u \omega(U))_{x} \mathrm{~d} x=\frac{9}{4} \int_{S^{1}} \rho^{4}(u \omega(U))_{x} \mathrm{~d} x .
$$

Hence

$$
\begin{aligned}
\mathscr{I}_{4} & \leq \frac{9}{4}\left\|(u \omega(U))_{x}\right\|_{L^{\infty}\left(S^{1}\right)}\|\rho\|_{L^{4}\left(S^{1}\right)}^{4} \\
& \leq \frac{9}{4} C_{4}\|\rho\|_{L^{4}\left(S^{1}\right)}^{4},
\end{aligned}
$$

by Lemma 4.15, Collecting the results (4.50), (4.56), (4.57) and (4.59), substituting into (4.49) we have

$$
\mathrm{d}_{t} F_{1}(\rho) \leq \mathscr{C}_{4} F_{1}(\rho)+\left\|\left(\omega(U)_{t}-U_{t}^{c}\right)_{x}\right\|_{L^{2}\left(S^{1}\right)}+\frac{1}{4}\left\|\omega(U)_{t}-U_{t}\right\|_{L^{4}\left(S^{1}\right)}^{4}+C_{a}^{2} C_{b}^{2}\left\|U_{t}^{d}\right\|_{V(h)}^{2} .
$$

The result follows from Gronwall's inequality.

Corollary 4.17 (Computable a posteriori bound - nonlinear case, $m=4$ ). Let the conditions of Theorem 4.16 hold. Then, with $e:=u-U$, for $t \in[0, T]$, and $m=4$ we have

$$
\begin{aligned}
& \frac{1}{2}\|e(t)\|_{\mathbb{V}_{q}}^{2}+\frac{1}{4}\|e(t)\|_{L^{4}\left(S^{1}\right)}^{4} \leq 2 \eta\left(U(t), g(t), H^{1}\left(S^{1}\right)\right)^{2} \\
& +8 \exp \left(\mathscr{C}_{4} t\right)\left(F_{1}(\rho(0))+\int_{0}^{t} 2 \eta\left(U_{t}(s), g_{s}(s), H^{1}\left(S^{1}\right)\right)^{2}\right. \\
& \left.+\left(2 C_{a}^{2} C_{b}^{2}\right)\left\|U_{s}^{d}(s)\right\|_{V(h)}^{2} \mathrm{~d} s\right) \\
& =: E_{s t}(U(t)) .
\end{aligned}
$$

Note that, by an abuse of notation, $E_{s t}$ is the computed quantity in the numerical results in Section 6 ,

Proof. This proof follows an equivalent argument to that made in the proof of Corollary 4.13 .

Remark 4.18. The bound for the point-wise in time $L^{m}$ error, $m=2,4$ appearing on the left-hand side of (4.30) and 4.61), is tight only for very short times. As we will observe in Section 5 on a uniform mesh of size $h \rightarrow 0$ the gradient term $\|u-U\|_{\mathbb{V}_{q}}=\mathrm{O}\left(h^{q}\right)$, while $\|(u-U)(t)\|_{L^{m}\left(S^{1}\right)}=\mathrm{O}\left(h^{q+1}\right)$.

\section{TEMPoral Discretisation AND NUMERICAL BENCHMARKING}

Practically, a fully discrete approximation scheme is required for implementation. For the readers convenience we will present an argument for designing a fully discrete scheme. We consider a time interval $[0, T]$ subdivided into a partition of $N$ consecutive adjacent sub-intervals whose endpoints are denoted $t_{0}=0<t_{1}<\ldots<t_{N}=T$. The $n$-th time-step is defined as $\tau_{n}:=t_{n+1}-t_{n}$. We will consistently use the shorthand $y^{n}(\cdot):=y\left(\cdot, t_{n}\right)$ for a generic time function $y$. We also denote $y^{n+\frac{1}{2}}:=\frac{1}{2}\left(y^{n}+y^{n+1}\right)$. 
We consider the temporal discretisation of (3.11) as follows: Given $U^{0}$, for $n \in[0, N-1]$ find $U^{n+1}$ such that

$$
\begin{aligned}
0 & =\int_{\mathbb{S}^{1}}\left(\frac{U^{n+1}-U^{n}}{\tau_{n}}+\mathscr{G}_{h}\left(V^{n+1}\right)\right) \Phi \mathrm{d} x \\
0 & =\int_{\mathbb{S}^{1}}\left(V^{n+1}+\frac{f\left(U^{n+1}\right)-f\left(U^{n}\right)}{U^{n+1}-U^{n}}\right) \Psi \mathrm{d} x+\mathscr{A}_{h}\left(U^{n+1 / 2}, \Psi\right) \quad \forall(\Phi, \Psi) \in \mathbb{V}_{q} \\
U^{0} & =\Pi^{0} u^{0}
\end{aligned}
$$

where $\Pi^{0}$ denotes the $L^{2}$ orthogonal projector into $\mathbb{V}_{q}$.

Theorem 5.1 (Conservativity of the fully discrete scheme). Let $\left\{U^{n}\right\}_{n=0}^{N}$ be the fully discrete scheme generated by (5.1), then we have that

$$
\int_{S^{1}} U^{n} \mathrm{~d} x=\int_{S^{1}} U^{0} \mathrm{~d} x \quad \forall n \in[0, N]
$$

and

$$
\frac{1}{2} \mathscr{A}_{h}\left(U^{n}, U^{n}\right)+\int_{S^{1}} f\left(U^{n}\right) \mathrm{d} x=\frac{1}{2} \mathscr{A}_{h}\left(U^{0}, U^{0}\right)+\int_{S^{1}} f\left(U^{0}\right) \mathrm{d} x \quad \forall n \in[0, N] .
$$

Proof. To show (5.2) it suffices to show that

$$
\int_{S^{1}} U^{n+1} \mathrm{~d} x=\int_{S^{1}} U^{n} \mathrm{~d} x
$$

and then the result follows inductively. To this end, choosing $\Phi=1$ in (5.1)

$$
\begin{aligned}
0 & =\int_{\mathbb{S}^{1}}\left(\frac{U^{n+1}-U^{n}}{\tau_{n}}+\mathscr{G}_{h}\left(V^{n+1}\right)\right) \mathrm{d} x \\
& =\int_{\mathbb{S}^{1}} \frac{U^{n+1}-U^{n}}{\tau_{n}} \mathrm{~d} x
\end{aligned}
$$

by the definition of $\mathscr{G}_{h}$. To see (5.3)

$$
\begin{aligned}
\frac{1}{2} \mathscr{A}_{h}\left(U^{n+1}, U^{n+1}\right)- & \frac{1}{2} \mathscr{A}_{h}\left(U^{n}, U^{n}\right)+\int_{S^{1}} f\left(U^{n+1}\right)-f\left(U^{n}\right) \mathrm{d} x \\
& =\mathscr{A}_{h}\left(U^{n+1}-U^{n}, U^{n+\frac{1}{2}}\right)+\int_{S^{1}} \frac{f\left(U^{n+1}\right)-f\left(U^{n}\right)}{U^{n+1}-U^{n}}\left(U^{n+1}-U^{n}\right) \mathrm{d} x \\
& =-\int_{S^{1}} V^{n+1}\left(U^{n+1}-U^{n}\right) \mathrm{d} x \\
& =\int_{S^{1}} \tau V^{n+1} \mathscr{G}_{h}\left(V^{n+1}\right) \mathrm{d} x=0
\end{aligned}
$$

using the second equation of (5.1) and the skew-symmetry of $\mathscr{G}_{h}$, concluding the proof.

Remark 5.2 (Structure of the temporal discretisation). The temporal discretisation given in (5.1) is not a Runge-Kutta method unless the problem is linear. It resembles a midpoint discretisation and is formally of second order, however the treatment of the non-linearity is different. Although construction of higher order methods is possible they become very complicated to write down so we will not press this point here. Further to the method of lines dG-difference scheme we propose, other discretisation methods are indeed possible. The spatial discretisation can be coupled to space-time Galerkin procedures, using, for example a continuous Galerkin method in time to guarantee conservativity. It is even possible to make use of hybrid dG-cG approaches to construct flexible adaptive space-time schemes making use of recovered elements [18].

Remark 5.3 (Conservation of other invariants). This discretisation does not lend itself to conservation of other invariants, for example even the quadratic invariant $F_{0}$ is not conserved under this scheme. A class of Runge-Kutta methods that are able to exactly conserve all quadratic invariants are the Gauss-Radau family, this is because they are symplectic. When one considers higher order invariants, it seems that schemes must be designed individually and there seems to be no class that can exactly conserve all.

\section{NumERICAL EXPERIMENTS}

In this section we illustrate the performance of the method proposed through a series of numerical experiments. The brunt of the computational work has been carried out using Firedrake [43. We employ a Gauss quadrature of order $4 q$, where $q$ is the degree of the finite element space, to minimise quadrature error introduced into the implementation. Indeed, at this degree all integrals are performed exactly with the exception of the projection of the initial condition. The nonlinear system of equations are then approximated using the PETSc [4,5] Newton line search method with a tolerance of $10^{-13}$ on each time step. A combination of Paraview and Matplotlib have been used as visualisation tools. For each 
benchmark test we fix the polynomial degree $q$ and compute a sequence of solutions with $h=h(i)=2^{-i}$ and $\tau=C h$ so temporal discretisation error is negligible. This is done for a sequence of refinement levels, $i=l, \ldots, L$. We have previously used $S^{1}$ as the unitary periodic domain. For our numerical experiments, we have scaled the domain to $[0,40]$ for computational convenience.

Definition 6.1 (Experimental order of convergence). Given two sequences a $(i)$ and $h(i) \searrow 0$ we define the experimental order of convergence $(E O C)$ to be the local slope of the $\log (a(i))$ vs. $\log (h(i))$ curve, i.e.,

$$
\operatorname{EOC}(a, h ; i)=\frac{\log \left(\frac{a_{i+1}}{a_{i}}\right)}{\log \left(\frac{h_{i+1}}{h_{i}}\right)} .
$$

Definition 6.2 (Effectivity Index). Given two sequences $a(i)$ and $b(i)$, the effectivity index is defined by ratio of the two, i.e.,

$$
\operatorname{EI}(a, b ; i)=\frac{b(i)}{a(i)} .
$$

In the sequel, we shall exclusively use the effectivity index where $b(i)$ is a sequence of a posteriori errors and a $(i)$ the error measured in the norm $\|\cdot\|_{\mathbb{V}_{q}}$.

Remark 6.3 (Numerical deviation in $F_{1}$ ). While the analysis shows that our scheme exactly preserves the energy over arbitrarily long time, the implementation relies on linear and nonlinear systems that inherently require further approximation. The result of this is that the energy may deviate locally up to the tolerance of the linear and nonlinear solvers which introduces the possibility of these errors propagating over time. In our numerical tests we focus on studying the global deviation in time, $F_{1}\left(U^{n}\right)-F_{1}\left(U^{0}\right)$, which includes any propagation arising from solver or precision errors.

Test 1: Conservativity and convergence of the linear scheme. We begin by examining the global deviation in invariants for the linear problem, i.e., when $f(u)=\frac{1}{2} u^{2}$. We observe, in Figure 1, that the both problems conserve the expected invariants.

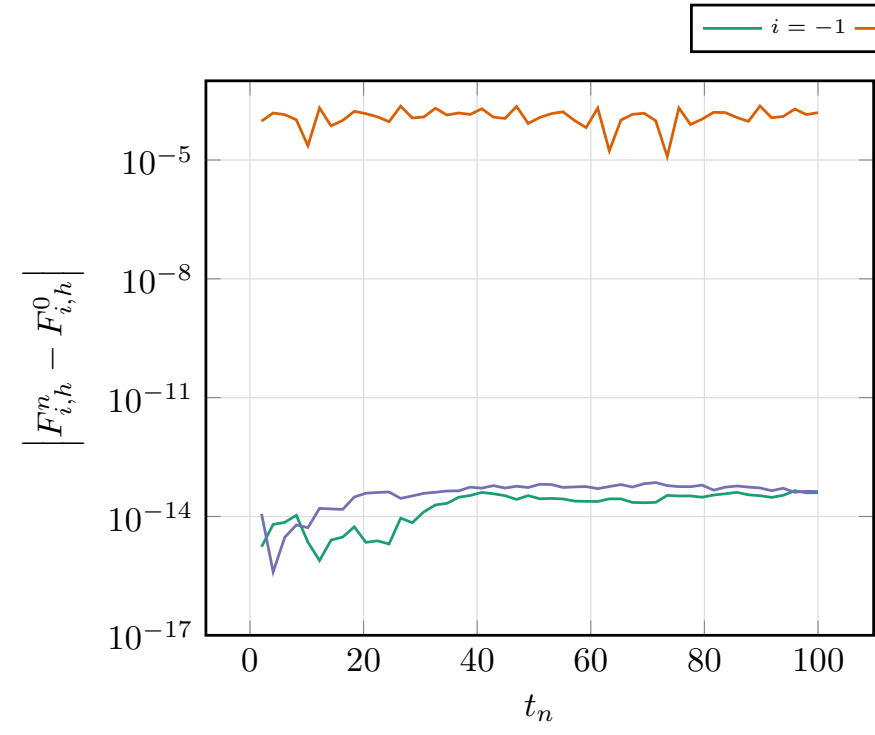

(a) $q=1$

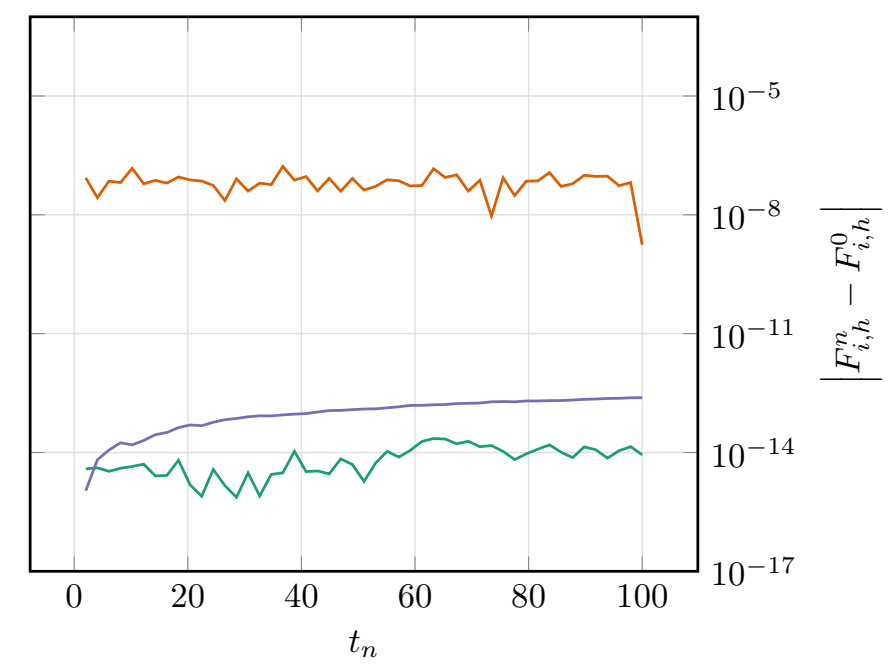

(b) $q=2$

Figure 1. The deviation in mass, momentum and energy with $T=100$ for the scheme (5.1) with $f(u)=\frac{1}{2} u^{2}$. The initial conditions are given by (2.15) where $l=1, C_{1}=1, C_{2}=0$, further we choose $\tau_{n}=0.2, h_{m}=0.5$ and vary the polynomial degree $q$. Notice that the deviation in mass and energy is below our solver tolerance of $10^{-13}$ for all $q$ with the deviation in momentum decreasing as we increase $q$.

We plot the experimental order of convergence for the linear problem in Figure 3 . We observe that the method convergences at the rate shown in the a priori bound (3.58), and the a posteriori error bound (4.30) behaves optimally. 


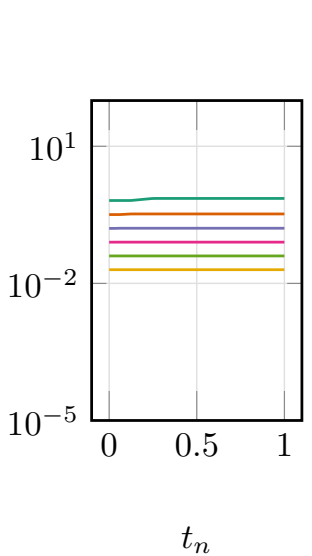

(a) $e$

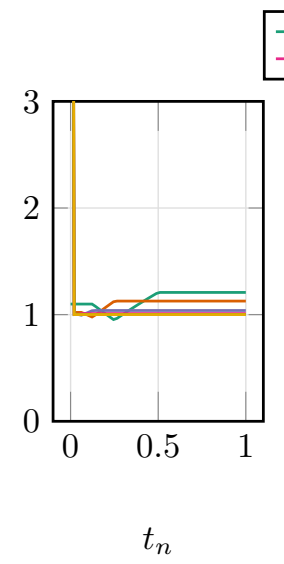

(b) $E O C(e)$

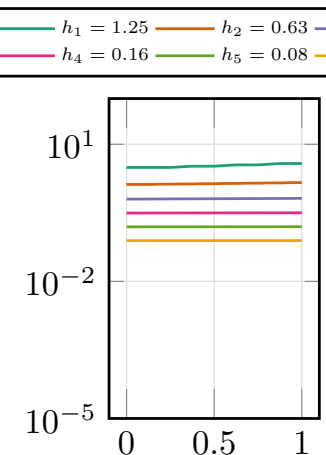

$t_{n}$

(c) $E_{s t}$

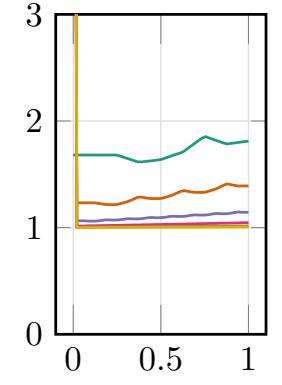

$t_{n}$

(d) $\operatorname{EOC}\left(E_{s t}\right)$

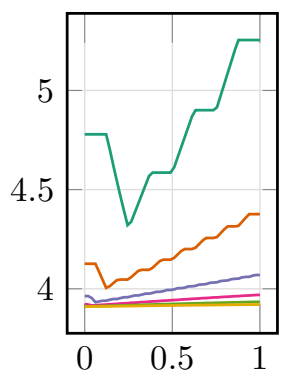

$t_{n}$

(e) $E I$

FIgURE 2. The error $e:=\|u-U\|_{\mathbb{V}_{q}}$ and the a posteriori estimator (4.37) denoted $E_{s t}$, and their associated experimental order of convergence with the corresponding exact solution (2.15) where $l=1, C_{1}=$ $1, C_{2}=0$ with polynomial degree $q=1$. We vary $h_{m}=0.1 \tau_{n}$, and observe the a priori bound (3.58) is attained for $q=1$, and that the a posteriori bound is optimal.

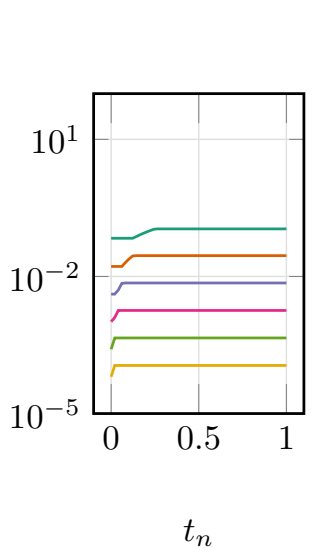

(a) $e$

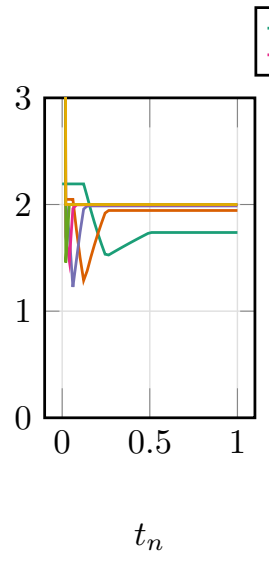

(b) $\operatorname{EOC}(e)$
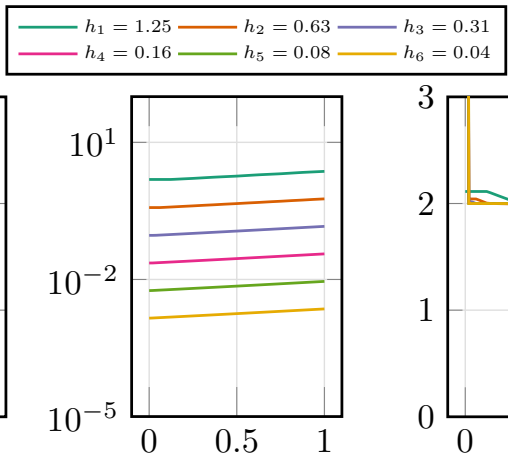

$t_{n}$

(c) $E_{\text {st }}$

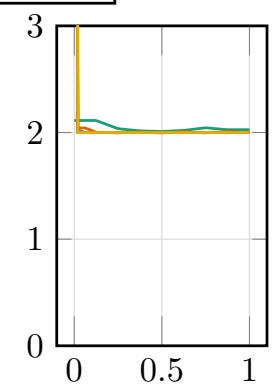

$t_{n}$

(d) $\operatorname{EOC}\left(E_{s t}\right)$

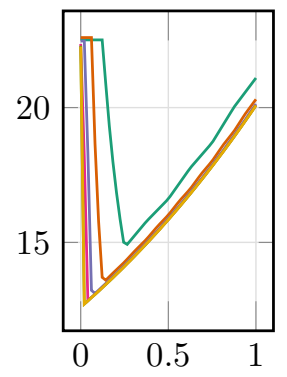

$t_{n}$

(e) $E I$

FIgURE 3. The error $e:=\|u-U\|_{\mathbb{V}_{q}}$ and the a posteriori estimator (4.37) denoted $E_{s t}$, and their associated experimental order of convergence with the corresponding exact solution (2.15) where $l=1, C_{1}=$ $1, C_{2}=0$ with polynomial degree $q=2$. We vary $h_{m}=0.1 \tau_{n}$, and observe the a priori bound (3.58) is attained for $q=1$, and that the a posteriori bound is optimal.

Test 2: Conservativity and convergence of the nonlinear scheme. Through examining the global deviation in invariants for the nonlinear problem $f(u)=\frac{1}{2} u^{4}$ we observe, in Figure 4, that the both problems conserve the expected invariants. 


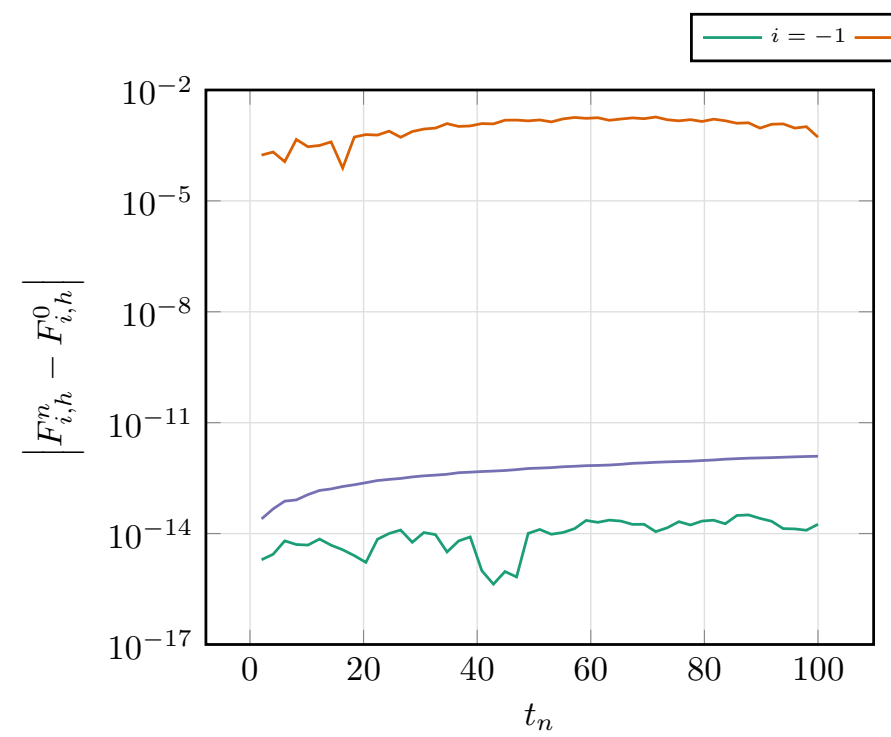

(a) $q=1$

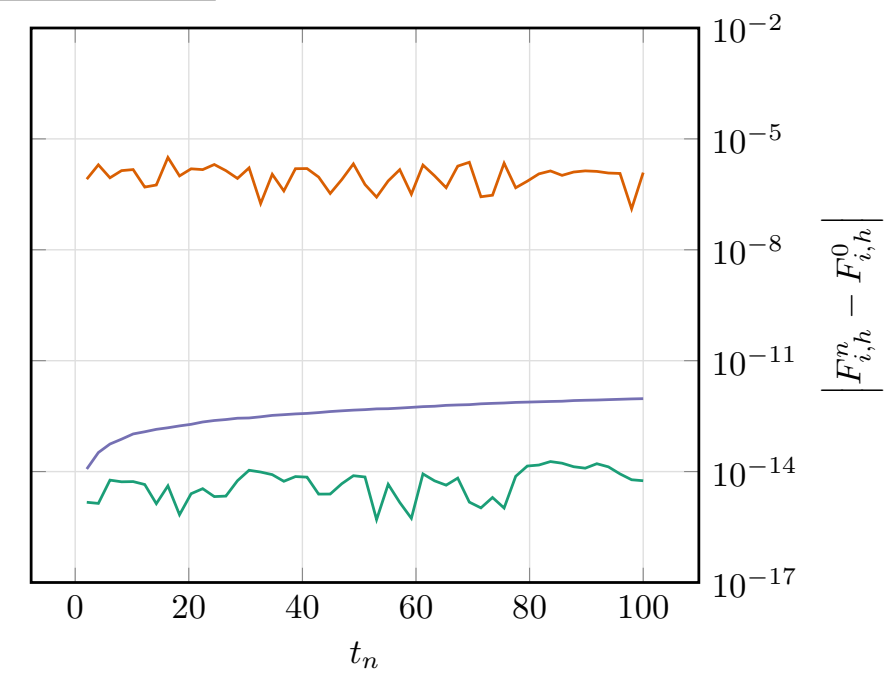

(b) $q=2$

Figure 4. The deviation in mass, momentum and energy with $T=100$ for the scheme (5.1) with $f(u)=\frac{1}{2} u^{4}$. The initial conditions are given by (2.19) with $k=0.9$. We additionally stretch our spatial interval to $x \in[0,16 K(k)]$, where $K(k)$ is the complete elliptic integral of the first kind, which numerically ensures that our solution is periodic up to a tolerance of $10^{-13}$. Further we choose $\tau_{n}=0.2, h_{m}=0.5$ and vary the polynomial degree $q$. The deviation in both mass and energy is below solver precision, with momentum decreasing as we increase $q$. We note that as we increase $q$ the momentum does not decrease as quickly as for the linear case.

In addition, we benchmark the nonlinear scheme against the exact solution (2.19) with $k=0.9$ over the stretched spatial domain $x \in[0,16 K(k)]$ (where $K(k)$ is the complete elliptic integral of the first kind) yielding Figure 6. We observe similar convergence rates to the linear case, satisfying the a posteriori error bound, in addition to indicating the existence of optimal a priori bounds.

\section{Conclusions And outlook}

In this work we have developed a novel discontinuous Galerkin scheme for a specific class of Hamiltonian problem. We have shown the method to be well posed and demonstrated that it inherits desirable conservative properties of the PDE.

Furthermore, we have conducted an a priori error analysis that shows the method is optimally convergent in the energy norm. This is not surprising as the method is deliberately designed to be conservative and the invariant induces the energy norm.

An a posteriori analysis was carried out for the semi discrete scheme that is very much in the spirit of the original elliptic reconstruction framework of [37. It is shown that we can make use of this framework to derive a posteriori bounds in the energy norm, this is different to the framework of [29] where a dispersive reconstruction was used to enable $L^{2}$ error control. An attractive feature of the analysis we present is that the bound holds irrespective of the underlying polynomial degree of the approximation scheme.

As an outlook we plan to make use of the a posteriori framework developed here to extend to fully discrete a posteriori bounds that are able to account for mesh adaptivity. This is a particularly subtle point as mesh change, when done in a naive way, can actually induce instabilities [7] although one can design adaptive schemes that ensure compatibility with the underlying Hamiltonian formulation of the problem [16, 40]. 


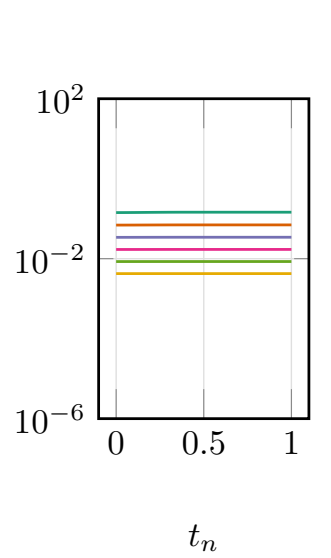

(a) $e$

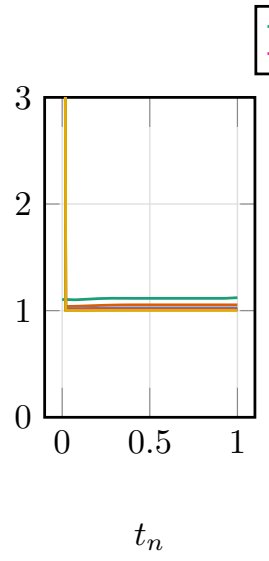

(b) $\operatorname{EOC}(e)$

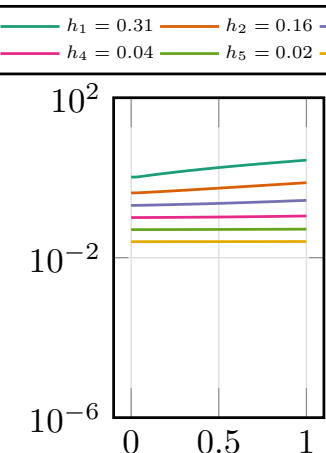

$t_{n}$

(c) $E_{s t}$

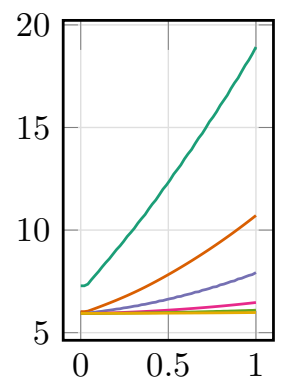

$t_{n}$

(e) $E I$

Figure 5. The error $e:=\|u-U\|_{\mathbb{V}_{q}}$ and the a posteriori estimator (4.46) denoted $E_{s t}$, and their associated experimental order of convergence using the solution (2.19) with $k=0.9$ and using polynomial degree $q=1$. We vary $h_{m}=0.1 \tau_{n}$, and observe that the a priori and a posteriori error bounds both converge optimally.

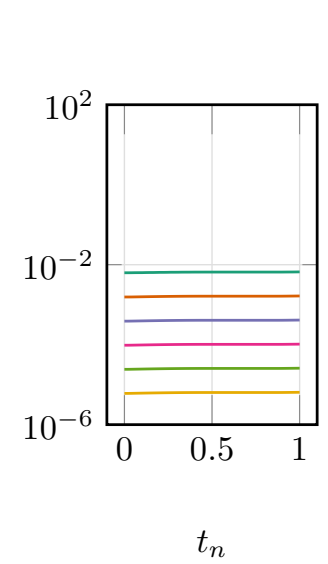

(a) $e$

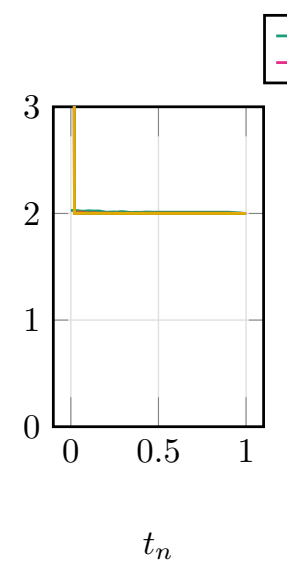

(b) $\operatorname{EOC}(e)$

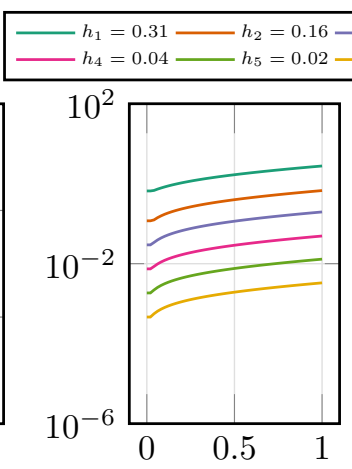

$t_{n}$

(c) $E_{s t}$

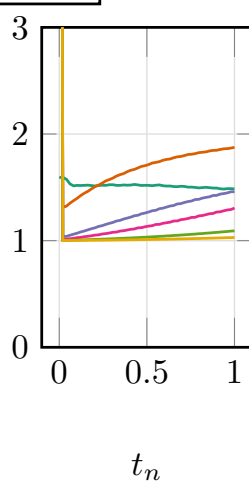

(d) $\operatorname{EOC}\left(E_{s t}\right)$ 


\section{REFERENCES}

[1] M. Ablowitz and P. Clarkson. Solitons, nonlinear evolution equations and inverse scattering, volume 149. Cambridge university press, 1991.

[2] M. Ainsworth and J. T. Oden. A posteriori error estimation in finite element analysis. Pure and Applied Mathematics (New York). Wiley-Interscience [John Wiley \& Sons], New York, 2000.

[3] U. M. Ascher and R. I. McLachlan. On symplectic and multisymplectic schemes for the KdV equation. J. Sci. Comput., 25(1-2):83-104, 2005.

[4] S. Balay, S. Abhyankar, M. F. Adams, J. Brown, P. Brune, K. Buschelman, L. Dalcin, V. Eijkhout, W. D. Gropp, D. Kaushik, M. G. Knepley, D. A. May, L. C. McInnes, R. T. Mills, T. Munson, K. Rupp, P. Sanan, B. F. Smith, S. Zampini, H. Zhang, and H. Zhang. PETSc users manual. Technical Report ANL-95/11 - Revision 3.9, Argonne National Laboratory, 2018.

[5] S. Balay, W. D. Gropp, L. C. McInnes, and B. F. Smith. Efficient management of parallelism in object oriented numerical software libraries. In E. Arge, A. M. Bruaset, and H. P. Langtangen, editors, Modern Software Tools in Scientific Computing, pages 163-202. Birkhäuser Press, 1997.

[6] R. E. Bank and J. Xu. Asymptotically exact a posteriori error estimators. II. General unstructured grids. SIAM J. Numer. Anal., 41(6):2313-2332 (electronic), 2003.

[7] E. Bänsch, F. Karakatsani, and C. Makridakis. The effect of mesh modification in time on the error control of fully discrete approximations for parabolic equations. Appl. Numer. Math., 67:35-63, 2013.

[8] S. Blanes and F. Casas. A Concise Introduction to Geometric Numerical Integration. CRC Press, 2016.

[9] O. Bokhove and P. Lynch. Air parcels and air particles: Hamiltonian dynamics. Nieuw Arch. Wiskd. (5), 8(2):100-106, 2007.

[10] J. L. Bona, H. Chen, O. Karakashian, and Y. Xing. Conservative, discontinuous Galerkin-methods for the generalized Korteweg-de Vries equation. Math. Comp., 82(283):1401-1432, 2013.

[11] E. Celledoni, V. Grimm, R. I. Mclachlan, D. I. Mclaren, D. O'Neale, B. Owren, and G. R. W. Quispel. Preserving energy resp. dissipation in numerical pdes using the "average vector field" method. J. Comput. Phys., 231(20):6770-6789, Aug. 2012.

[12] E. Celledoni and J. Jackaman. Discrete conservation laws for finite element discretisations of multisymplectic PDEs. arXiv preprint arXiv:2009.00102, 2020.

[13] B. Deconinck and M. Nivala. The stability analysis of the periodic traveling wave solutions of the mkdv equation. Studies in Applied Mathematics, 126(1):17-48, 2011.

[14] A. Dedner, J. Giesselmann, T. Pryer, and J. K. Ryan. Residual estimates for post-processors in elliptic problems. To appear in Springer Journal of Scientific Computing, 2021.

[15] T. A. Driscoll, F. Bornemann, and L. N. Trefethen. The chebop system for automatic solution of differential equations. BIT Numerical Mathematics, 48(4):701-723, 2008.

[16] S. l. Eidnes, B. Owren, and T. r. Ringholm. Adaptive energy preserving methods for partial differential equations. Adv. Comput. Math., 44(3):815-839, 2018.

[17] A. Ern and J.-L. Guermond. Theory and practice of finite elements, volume 159 of Applied Mathematical Sciences. Springer-Verlag, New York, 2004.

[18] E. H. Georgoulis and T. Pryer. Recovered finite element methods. Comput. Methods Appl. Mech. Engrg., 332:303-324, 2018.

[19] J. Giesselmann, C. Makridakis, and T. Pryer. Energy consistent discontinuous Galerkin methods for the Navier-Stokes-Korteweg system. Math. Comp., 83(289):2071-2099, 2014.

[20] J. Giesselmann, C. Makridakis, and T. Pryer. A posteriori analysis of discontinuous Galerkin schemes for systems of hyperbolic conservation laws. SIAM J. Numer. Anal., 53(3):1280-1303, 2015.

[21] J. Giesselmann and T. Pryer. Energy consistent discontinuous Galerkin methods for a quasi-incompressible diffuse two phase flow model. ESAIM Math. Model. Numer. Anal., 49(1):275-301, 2015.

[22] J. Giesselmann and T. Pryer. Reduced relative entropy techniques for a posteriori analysis of multiphase problems in elastodynamics. IMA Journal of Numerical Analysis, 36(4):1685-1714, 2015.

[23] J. Giesselmann and T. Pryer. Reduced relative entropy techniques for a priori analysis of multiphase problems in elastodynamics. BIT, 56(1):99-127, 2016.

[24] J. Giesselmann and T. Pryer. A posteriori analysis for dynamic model adaptation in convection-dominated problems. Mathematical Models and Methods in Applied Sciences, 27(13):2381-2423, 2017.

[25] E. Hairer, C. Lubich, and G. Wanner. Geometric numerical integration, volume 31 of Springer Series in Computational Mathematics. Springer-Verlag, Berlin, second edition, 2006. Structure-preserving algorithms for ordinary differential equations.

[26] P. Houston, I. Perugia, and D. Schotzau. Mixed discontinuous galerkin approximation of the Maxwell operator. SIAM Journal on Numerical Analysis, 42(1):434-459, 2004.

[27] J. Jackaman, G. Papamikos, and T. Pryer. The design of conservative finite element discretisations for the vectorial modified KdV equation. Applied Numerical Mathematics, 137:230-251, 2019.

[28] J. Jackaman and T. Pryer. Quasinorms in semilinear elliptic problems. In Boundary and Interior Layers, Computational and Asymptotic Methods BAIL 2018, pages 183-200. Springer, 2020.

[29] O. Karakashian and C. Makridakis. A posteriori error estimates for discontinuous Galerkin methods for the generalized Korteweg-de Vries equation. Math. Comp., 84(293):1145-1167, 2015.

[30] T. Kato. Quasi-linear equations of evolution, with applications to partial differential equations. In Spectral theory and differential equations, pages 25-70. Springer, 1975.

[31] E. Kesici, B. Pelloni, T. Pryer, and D. Smith. A numerical implementation of the unified Fokas transform for evolution problems on a finite interval. European Journal of Applied Mathematics, 29(3):543-567, 2018.

[32] O. Lakkis and C. Makridakis. Elliptic reconstruction and a posteriori error estimates for fully discrete linear parabolic problems. Math. Comp., 75(256):1627-1658 (electronic), 2006.

[33] O. Lakkis, C. Makridakis, and T. Pryer. A comparison of duality and energy a posteriori estimates for $L^{\infty}\left(L^{2}\right)$ in parabolic problems. Mathematics of Computation, 84(294):1537-1569, 2015.

[34] O. Lakkis and T. Pryer. Gradient recovery in adaptive finite-element methods for parabolic problems. IMA Journal of Numerical Analysis, 32(1):246-278, 2011. 
[35] B. Leimkuhler and S. Reich. Simulating Hamiltonian dynamics, volume 14 of Cambridge Monographs on Applied and Computational Mathematics. Cambridge University Press, Cambridge, 2004.

[36] F. Magri. A simple model of the integrable Hamiltonian equation. Journal of Mathematical Physics, 19(5):1156-1162, 1978.

[37] C. Makridakis and R. H. Nochetto. Elliptic reconstruction and a posteriori error estimates for parabolic problems. SIAM J. Numer. Anal., 41(4):1585-1594 (electronic), 2003.

[38] C. Makridakis and R. H. Nochetto. A posteriori error analysis for higher order dissipative methods for evolution problems. Numer. Math., 104(4):489-514, 2006.

[39] R. I. McLachlan and A. Stern. Multisymplecticity of hybridizable discontinuous Galerkin methods. Found. Comput. Math., 20(1):35-69, 2020.

[40] Y. Miyatake and T. Matsuo. A note on the adaptive conservative/dissipative discretization for evolutionary partial differential equations. Journal of Computational and Applied Mathematics, 274:79-87, 2015.

[41] P. Müller, C. Garrett, and A. Osborne. Rogue waves. Oceanography, 18(3):66-75, 2005.

[42] J. A. Pava, J. L. Bona, M. Scialom, et al. Stability of cnoidal waves. Advances in Differential Equations, 11(12):1321-1374, 2006.

[43] F. Rathgeber, D. A. Ham, L. Mitchell, M. Lange, F. Luporini, A. T. T. McRae, G.-T. Bercea, G. R. Markall, and P. H. J. Kelly. Firedrake: automating the finite element method by composing abstractions. ACM Trans. Math. Software, 43(3):Art. 24, $27,2017$.

[44] I. Roulstone and J. Norbury. A Hamiltonian structure with contact geometry for the semi-geostrophic equations. J. Fluid Mech., 272:211233, 1994.

[45] R. Winther. A conservative finite element method for the Korteweg-de Vries equation. Math. Comp., 34(149):23-43, 1980.

[46] Y. Xu and C.-W. Shu. Error estimates of the semi-discrete local discontinuous Galerkin method for nonlinear convection-diffusion and KdV equations. Comput. Methods Appl. Mech. Engrg., 196(37-40):3805-3822, 2007.

[47] J. Yan and C.-W. Shu. A local discontinuous Galerkin method for KdV type equations. SIAM J. Numer. Anal., 40(2):769-791 (electronic), 2002.

[48] P. F. Zhao and M. Z. Qin. Multisymplectic geometry and multisymplectic Preissmann scheme for the KdV equation. J. Phys. A, 33(18):3613-3626, 2000.

[49] O. C. Zienkiewicz and J. Z. Zhu. A simple error estimator and adaptive procedure for practical engineering analysis. Internat. J. Numer. Methods Engrg., 24(2):337-357, 1987.

James Jackaman Department of Mathematics and Statistics, Memorial University of Newfoundland, St. John's, NL, A1C 5S7, CANADA jjackaman@mun.ca

Tristan Pryer Department of Mathematical Sciences, University of Bath, Bath BA2 7AY, UK tmp38@bath.ac.uk 